\title{
Steigerung der Lern- und Innovationsfähigkeit von Unternehmen und Organisationen
}

\section{Inhalt}

1 Problemstellung: Häufige Innovationsbarrieren?

2 Lern- und Innovationsfähigkeit von Organisationen: Konzeptionelle Grundlagen ....................... 3

3 Rahmenkonzept zur Steigerung der Lern- und Innovationsfähigkeit von Organisationen .............6

4 Zentrale Handlungsfelder zur Erhöhung der Lern- und Innovationsfähigkeit von Organisationen .. 8

4.1 Individuelle Kompetenzentwicklung: Förderung innovativen Verhaltens ..........................8 8

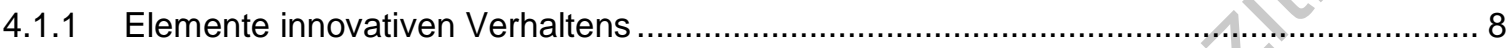

4.1.2 Wissensarten: Arbeitsprozesswissen und Kompetenzen.................................... 8

4.1.3 Erfolgsfaktoren für innovatives Verhalten von Organisationsmitgliedern ...................... 10

4.2 Lern- und innovationsorientierte Führungskräfteentwicklung ...................................... 12

4.2.1 Führungskompetenzen als Voraussetzung der Förderung innovativen Verhaltens ......... 12

4.2.2 Führungskräfte als Personal- und Kompetenzentwickler ........................................ 13

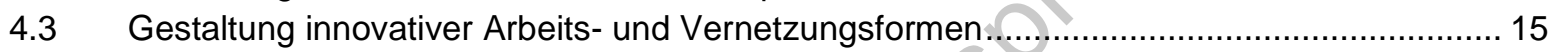

4.3.1 Kompetenzentwicklung: Verzahnung von formellem und informellen Lernen ................. 15

4.3.2 "Neue“ Vernetzungsformen zwischen Mitarbeitenden, Kunden und Partnern ................ 16

4.4 Gestaltung lern- und innovationsförderlicher Rahmenbedingungen ........................... 18

4.4.1 Rahmenbedingungen: Anreizsysteme und Infrastrukturen gestalten ......................... 18

4.4.2 Lernkulturanalyse als kontinuierliche OE Maßnahme ....................................... 20

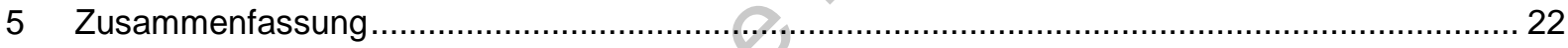

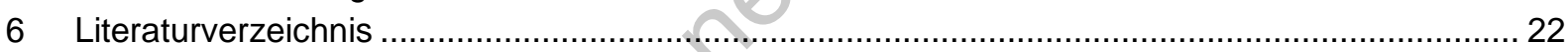

\section{Problemstellung: Häufige Innovationsbarrieren?}

Business Innovation bedarf einer organisationalen Verankerung im Unternehmen, was entsprechende Kulturen, Strukturen und eine Strategie umfasst. In diesem Beitrag wird ein Rahmenmodell vorgestellt, das auf die Gestaltung dieser auf der Grundlage von vier Handlungsfeldern eingeht.

Die Fähigkeit, Innovationen umzusetzen, wird für Organisationen aufgrund zunehmender Wettbewerbsdynamiken immer bedeutender. Um Innovationspotenziale auszuschöpfen, nehmen Innovationen aktuell ein breites Spektrum in Unternehmen ein - damit einher geht ein neues, „modernes Innovationsverständnis“ (Reinmann, 2013, S. 4). War früher ausschließlich die F\&EAbteilung für Innovationen zuständig, wird heute neben darauf spezialisierten Rollenprofilen Innovation in der Breite gefordert: Das gesamte Unternehmen und jedes seiner Mitglieder ist letztendlich für Innovationen verantwortlich, wobei davon ausgegangen wird, dass „Gruppenarbeit und Teamgeist eher zum Erfolg führen als individuell-einsames Nachdenken und Ellenbogenmentalität“" (Reinmann, 2013, S. 4). Es geht dabei nicht zwingend um radikale Innovationen in einem traditionellen Verständnis, vielmehr auch um weniger auffällige inkrementelle Innovationen sowie damit verbunden um Erhalt und Optimierung von Bestehendem, „sofern damit neue und nachhaltige Veränderungen in Gang gesetzt werden“ (Reinmann, 2013, S. 4), die zu neuen Prozessen und Servicequalität führen.

Doch wie kann die Innovationsfähigkeit einer Organisation gewährleistet bzw. gefördert werden? In Wissenschaft und Praxis ist man sich einig: „Innovationsfähigkeit setzt die Fähigkeit zum permanenten 
Lernen voraus“ (Weissenberger-Eibl, 2010, S. 23). Lernen stellt also eine Voraussetzung für Innovationen dar. In diesem Zusammenhang stehen auch die Begriffe „organisationales Lernen“ mit einer starken wissenschaftlichen Verankerung sowie „lernende Organisation“ als Management-Ansatz aus der Praktiker-Literatur. Es existieren hierzu zahlreiche Definitionen, grundsätzlich ist dabei eine anpassungs- bzw. entwicklungsfähige Organisation gemeint. Organisationen lernen allerdings nur, d.h. entwickeln sich weiter bzw. sind innovationsfähig, wenn die einzelnen Mitglieder lernen. Umgekehrt führt aber individuelles Lernen nicht zwingend zum Lernen der Organisation. (Seufert, 2013, S. 59-60)

Dem Lernen und damit verbundenen Veränderungen können jedoch auch Hindernisse entgegenstehen: Auf der Ebene der Mitarbeitenden bspw. durch den Wunsch nach einer Komfortzone oder durch individuelle Präferenzen; auf der Ebene der Organisation bspw. indem ein „Einschleifen von Routinen“ (Wilkesmann, 1999, S. 10) und damit reines Anpassungslernen statt neuen Handlungsmöglichkeiten gefördert wird. „Routinemuster des Verhaltens“ bringen keine Innovationen hervor (Haller, 2003, S. 195, nach DeBono, 1997, S. 23), es braucht vielmehr "'learning the new" and being in the flow of new thinking, new ideas" (Hart, 2013, S. 11).

Organisationale Hindernisse können sich schnell auf individuelles Lernen und damit auf die Innovationsfähigkeit der Organisation negativ auswirken. In Anlehnung an das St.Galler Management Modell (nach Rüegg-Stürm, 2003) können Innovationshindernisse in der Strategie, in den Strukturen und in der Kultur der Organisation begründet sein. Bspw. können „Scheuklappen“ durch Strukturen und Kulturen entstehen, ein „Korsett“ kann gegeben sein durch die Strategie und Kulturen und eine „Routine“, die auf Effizienz ausgerichtet ist und Komfortzonen unterstützt, kann durch Strukturen und Kulturen bedingt sein. Die folgende Abbildung stellt diese organisationalen Innovationsbarrieren dar.

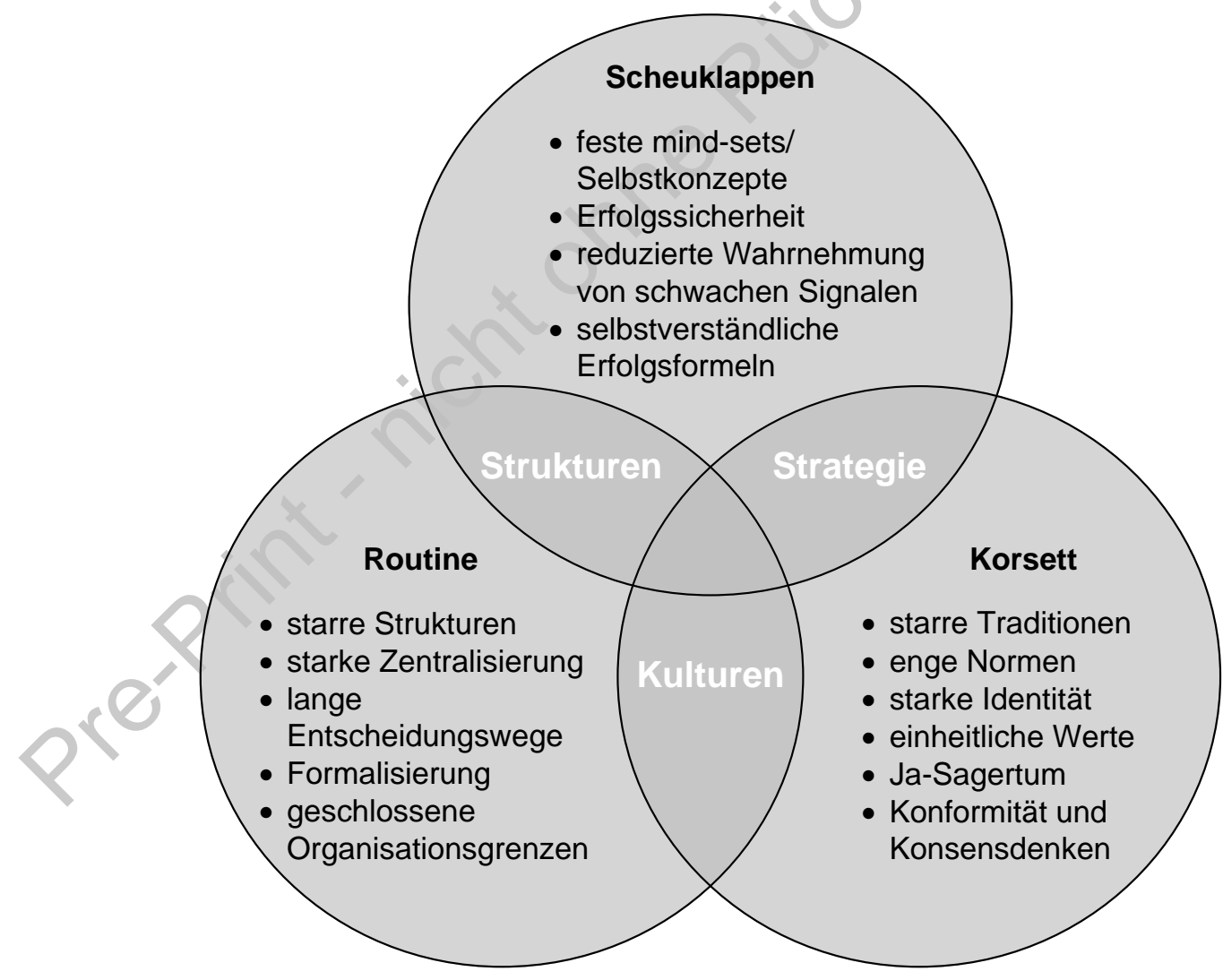

Abbildung 1: Organisationale Innovationsbarrieren: Scheuklappen, Routine und Korsett (in Anlehnung an Hartmann, Brentel \& Rohn, 2006, S. 26)

Dieser Beitrag geht aus der Perspektive des organisationalen Bildungsmanagements der Frage nach, wie die Entwicklungsfähigkeit von Organisationen erhöht werden kann. Bildungsmanagement wird hier 
verstanden als dynamischer und kontinuierlicher Prozess, der im Endergebnis die Lern- und Innovationsfähigkeit von Individuen und der Organisation erhöht. Da Innovationsprozesse Lernprozesse voraussetzen, erfordert die Entwicklung von Innovationen somit Lernen der Organisationsmitglieder und der Organisation insgesamt. Im Fokus steht in den weiteren Ausführungen die Überwindung von Barrieren, die Lernen als Voraussetzung von Innovation negativ beeinträchtigen können. Einen Ansatz hierzu bietet die systematische Verzahnung von individuellem und organisationalem Lernen und damit die Verbindung von Personal- und Organisationsentwicklung, in dessen Rahmen die Gestaltung von Lernen in der Organisation didaktisch und managementtheoretisch abzustimmen ist.

\section{Lern- und Innovationsfähigkeit von Organisationen: Konzeptionelle Grundlagen}

Die Entwicklungsfähigkeit von Organisationen gewinnt zunehmend an Bedeutung und führt zur Implementierung von Organisationsformen, Führungskonzepten und Managementsystemen, die verstärkt auf Selbstorganisation der Mitarbeitenden setzen (vgl. u.a. Dehnbostel \& Pätzold, 2004; Wunderer, 2003). Hierbei kommt dem Lernen der einzelnen Mitarbeitenden ebenso wie der Entwicklung der Organisation insgesamt eine große Bedeutung zu, die mit der Institutionalisierung lern- und innovationsförderlicher Strukturen sowie Kulturen einhergeht.

Wie eingangs angeführt, ist die Lernfähigkeit einer Organisation Voraussetzung für die Innovationsfähigkeit einer Organisation - insbesondere was die Entwicklungs- und Anpassungsfähigkeit an veränderte Umweltbedingungen betrifft. Dabei ist Lernen klar auf Innovationen ausgerichtet: „Eine Organisation ist nicht nur zweckneutral lernfähig, sondern lernt im Endergebnis, wenn Innovationen bzw. das innovative Lösen von Problemen als Resultat entstehen" (Seufert, 2013, S. 58). Auch Hartmann et al. (2006) sehen die enge Verbindung von Lernen und Innovationen in der Herausforderung, „dass neue, situative und kreative Lösungen für eine bestimmte Problematik gefunden bzw. erfunden werden müssen. Die Fähigkeit zur Innovation hängt in großem Masse vom Vermögen ab, neue Ideen denken und umsetzen, bisherige Erfahrungen reflektieren und nicht zuletzt begründete Schlussfolgerungen ziehen zu können; Faktoren, die das organisationale Lernen begünstigen, sind auch für die Innovationsfähigkeit zentral. Die Übernahme und Anwendung neuer Ideen, Verfahren und Techniken erfordern Lernen aus Erfahrungen, Kreativität, Interpretationsund Entscheidungskompetenz auf der Basis valider Informationen ..." (2006, S. 20). Damit wird schon deutlich, dass die einzelnen Mitarbeitenden umfassende Handlungskompetenzen benötigen, um lernund innovationsfähig zu sein. Der Erwerb von solchen Kompetenzen in veränderten Umweltbedingungen sind sowohl auf Prozesse der Kompetenzentwicklung als auch Verbesserungsund Innovationsprozesse in einer Organisation ausgerichtet.

Innovationsfähigkeit bedeutet daher (Dehnbostel \& Pätzold, 2004, S. 23):

- für die Individuen: Kontinuierliches und reflexives Lernen in der Arbeit,

- für die Organisation: Ermöglichung und Realisierung von Innovationen.

Letzteres beinhaltet das Bereitstellen einer lernförderlichen Strategie, Strukturen und Kulturen, denn „ohne Experimentieren und aus Fehlern lernen zu können, d.h. eine ausgeprägte Fehler- und Kritikkultur zu praktizieren, Erfahrungen reflektieren zu können, grundlegende Normen und vermeintliche Gewissheiten hinterfragen und neu denken zu können, können Innovationen kaum produziert werden. Dies gilt für die organisationale und individuelle Ebene gleichermaßen." (Hartmann et al., 2006, S. 21)

Im Zusammenhang mit Innovationen nimmt die Nichtimitierbarkeit einer Organisation eine bedeutende Rolle ein. Damit ist das Bündel an Ressourcen gemeint, über das ein Unternehmen verfügt und das nicht einfach durch andere Unternehmen imitierbar ist: „Die Nichtimitierbarkeit nimmt dabei die wichtigste Rolle ein. Nicht oder nur schwer imitierbar sind vor allem solche Ressourcen, die nicht 
gekauft, sondern in langwierigen Prozessen im Unternehmen selbst erzeugt werden" (Osterloh, 2001, S. 125). Ebenso beschreibt Rüegg-Stürm (1998): „Es ist nahezu unmöglich, solche

Entwicklungsprozesse zu kopieren oder nachzuahmen, sie weisen vielmehr einen sehr hohen, zeitlich außerordentlich stabilen Imitationsschutz auf“ (Rüegg-Stürm, 1998, S. 258). Auf der Ebene der Mitarbeitenden stellt die intrinsische Arbeitsmotivation ein vergleichbar schwer zu imitierendes Merkmal dar (vgl. Erpenbeck 2004, S. 44). Dabei sind für die Mitarbeitenden Erfahrungswerte und Werterfahrungen (Erpenbeck, 2004, S. 43), die sie durch ihr berufliches Handeln machen können und die die Einmaligkeit eines beruflichen HandeInden ausmachen, bedeutend. Entsprechend ist es für das Bildungsmanagement wichtig, an Internalisierungs- (von Werten) und Sozialisationsprozessen innerhalb der Organisation mitzuwirken, um so die Entwicklung von entsprechenden organisationsspezifischen Werten, Normen, individuellen Emotionen und intrinsischer Motivationen der Mitarbeitenden sowie die gemeinsame Verständigung über solche Werte zu unterstützen. Die nachfolgende Abbildung veranschaulicht vier Ebenen organisationalen Lernens, die diese Überlegungen aufgreifen.

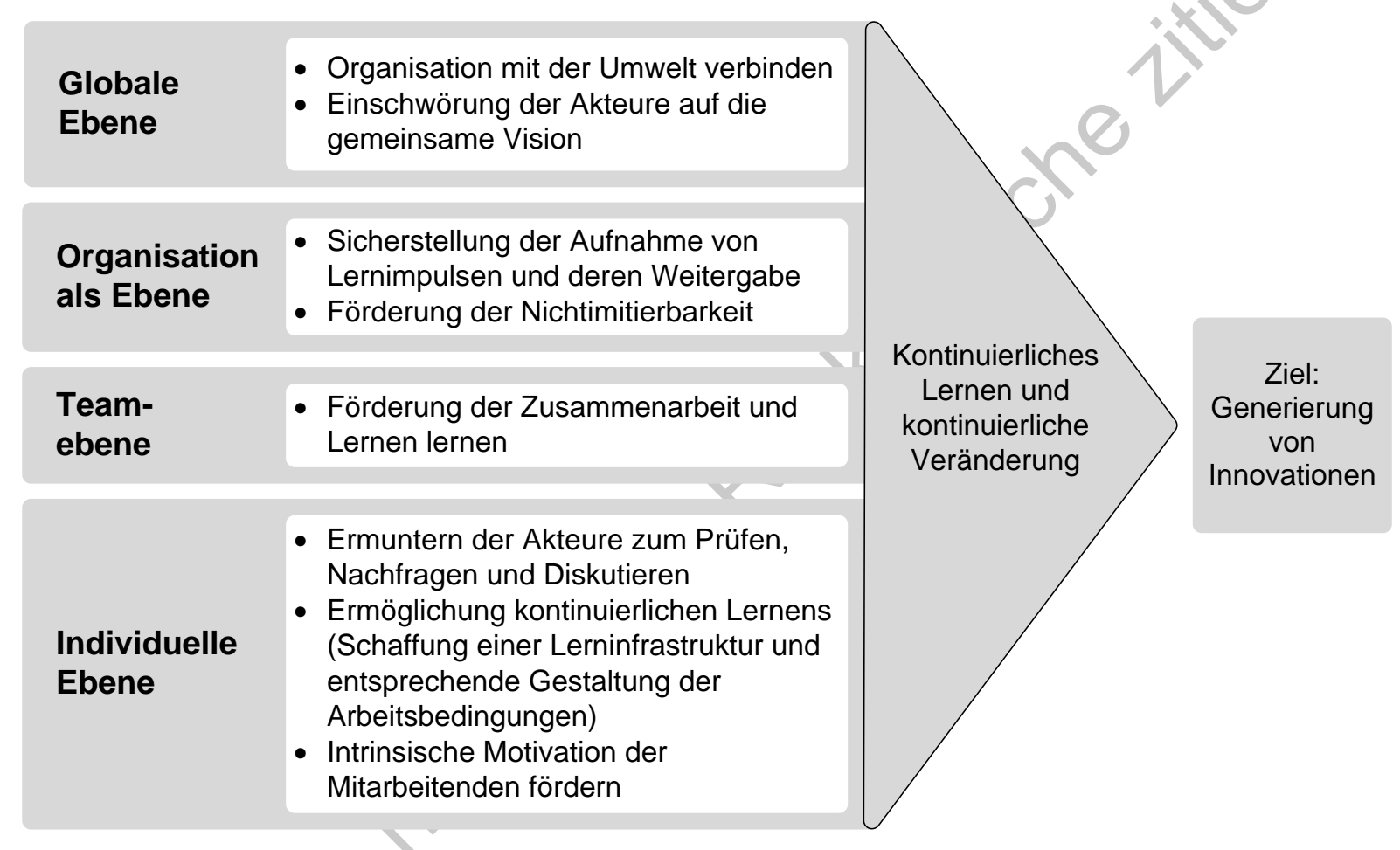

Abbildung 2: Vier Ebenen organisationalen Lernens

Für die Innovationsfähigkeit bzw. „das innovative Lösen von Problemen“ (Seufert, 2013, S. 58) einer Organisation sind das organisationale und das individuelle Lernen grundlegend. Die Organisation handelt und lernt dabei durch ihre Mitglieder. „Die Organisation konstituiert sich aus der Interaktion und Kommunikation der Individuen ..., mit jeweils einer individuellen Wissensbasis. Im Zuge der Interaktion und Kommunikation bildet sich eine gemeinsame, organisatorische Wissensbasis, die historisch bedingt ist. Handeln Individuen als Mitglieder der Organisation, d.h. nicht mehr im ausschließlich eigenen Kontext, ist ihr Verhalten auch immer von der organisatorischen Wissensbasis, insbesondere von kollektiv geltenden Handlungsstrategien, Normen, Standards und Weltsichten geprägt." (Haller, 2003, S. 399) Durch Interpretation von Reaktionen auf Verhalten können Individuen lernen und ihre Wissensbasis verändern. Durch Kommunikation und Interaktion können diese Lernerfahrungen in die organisationale Wissensbasis einfließen, woraufhin wieder Reaktionen folgen.

Soweit zum Zusammenhang von individuellem und organisationalem Lernen. Eine der bekanntesten Theorien für organisationales Lernen ist die von Argyris und Schön (1999), welche die nachfolgende Tabelle verdeutlicht. Die Autoren unterscheiden drei Arten bzw. Stufen organisationalen Lernens, die sich unterschiedlich auf Innovationsprozesse auswirken: 


\begin{tabular}{|c|c|c|}
\hline Lernstufe & Erläuterung & $\begin{array}{l}\text { Potenzial für } \\
\text { Innovationsprozesse }\end{array}$ \\
\hline $\begin{array}{l}\text { Single-Loop- } \\
\text { Learning: } \\
\text { Anpassungs- } \\
\text { lernen }\end{array}$ & $\begin{array}{l}\text { - verändertes Verhalten im Rahmen des } \\
\text { bisherigen Handlungsrepertoires, } \\
\text { - zielt auf Verbesserung und } \\
\text { Optimierung (Seufert, 2013, S. 58), } \\
\text { - findet statt, wenn Abweichungen zu } \\
\text { vorgegebenen Zielen oder Standards } \\
\text { auftreten und das Verhalten zu } \\
\text { dessen Erreichung angepasst wird, } \\
\text { - erfolgt passiv und „ist nicht in der } \\
\text { Lage, die Problemlösungskapazität } \\
\text { einer Organisation zu erhöhen“ } \\
\text { (Haller, 2003, S. 401). }\end{array}$ & $\begin{array}{l}\text { - kann bestenfalls einzelne Schritte } \\
\text { in einem spezifischen } \\
\text { Innovationsprojekt mit } \\
\text { abgegrenzten Vorgängen und } \\
\text { vorgegebenen Zielen und } \\
\text { Maßnahmen ermöglichen, da es } \\
\text { nur punktuelle } \\
\text { Verhaltensänderung umfasst, } \\
\text { - Effizienzverbesserung. }\end{array}$ \\
\hline $\begin{array}{l}\text { Double-Loop- } \\
\text { Learning: } \\
\text { Veränderungs- } \\
\text { lernen }\end{array}$ & $\begin{array}{l}\text { - führt zum Verlernen alter und Erlernen } \\
\text { neuer Verhaltensweisen, indem alte } \\
\text { als nicht ausreichend funktionierend } \\
\text { erkannt werden (Haller, 2003, S. 401), } \\
\text { - findet statt durch Hinterfragen eigener } \\
\text { Normen und beinhaltet die } \\
\text { Veränderung der Ziele und Standards } \\
\text { sowie mentaler Modelle, } \\
\text { - ist ebenfalls reaktiv, beinhaltet aber } \\
\text { bereits die Erweiterung des } \\
\text { Handlungsrepertoires (Seufert, 2013, } \\
\text { S. 58). }\end{array}$ & $\begin{array}{l}\text { - richtet sich auf ein spezifisches } \\
\text { Innovationsprojekt und nicht auf } \\
\text { die Entwicklung von Innovation } \\
\text { (wie Single-Loop-Learning), } \\
\text { - Ziele und Handlungsstrategien } \\
\text { können jedoch verändert werden } \\
\text { und zur höheren Effektivität eines } \\
\text { Innovationsprozesses beitragen. }\end{array}$ \\
\hline $\begin{array}{l}\text { Deutero-Learning: } \\
\text { Prozesslernen }\end{array}$ & $\begin{array}{l}\text { - macht den Lernprozess selbst zum } \\
\text { Lerngegenstand, wodurch die } \\
\text { Organisation fähig wird, diese auf } \\
\text { einer Meta-Ebene zu reflektieren } \\
\text { (Seufert, 2013, S. 58), } \\
\text { - Wissen über das Lernen (basierend } \\
\text { auf Erfahrungen der niedrigeren } \\
\text { Stufen) der Organisation wird } \\
\text { verändert, was zur Verbesserung der } \\
\text { Lernfähigkeit führt und in } \\
\text { Veränderungsprozessen der } \\
\text { Organisation mündet (Argyris \& } \\
\text { Schön, 1978, S. 4), } \\
\text { - alle Mitglieder der Organisation sind } \\
\text { Träger dieses Lernprozesses, } \\
\text { - stellt proaktives Lernen dar. }\end{array}$ & $\begin{array}{l}\text { - ermöglicht die Generierung von } \\
\text { Innovation, } \\
\text { - generelle Innovationsfähigkeit ist } \\
\text { losgelöst von spezifischen } \\
\text { Innovationsprojekten, } \\
\text { - auf dieser Ebene kann reflektiert } \\
\text { werden, inwiefern Single-, } \\
\text { Double-Loop- oder Deutero- } \\
\text { Learning notwendig ist; dies ist } \\
\text { bedeutend, da Unsicherheit und } \\
\text { Dynamik im organisationalen } \\
\text { Umfeld u.U. eine kontinuierliche } \\
\text { Veränderung des Verhaltens aller } \\
\text { Mitglieder erfordern. }\end{array}$ \\
\hline
\end{tabular}

Tabelle 1: Drei Stufen organisationalen Lernens und Potenzial für Innovationsprozesse

Auf der höchsten Stufe, der Stufe des Prozesslernens, wird Innovation also im Unternehmen institutionalisiert (Haller, 2003, S. 403), was auch „die Identifikation und Beseitigung von Lernbarrieren bzw. Barrieren zur Verhaltensänderung“ (Haller, 2003, S. 402) erfordert. Dafür müssen die Individuen und die Organisation als Kollektiv, „um den Prozess des Lernens selbst zu hinterfragen, ... (sich) in selbstreflexiver Weise mit dem eigenen Verhalten auseinandersetzen“ (Haller, 2003, S. 403, nach Geissler, 1994, S. 96). 
An diesen Ansatz anschlussfähig ist das Modell der fünf Disziplinen einer lernenden Organisation nach Senge (1996). Dieses stellt das Lernen der einzelnen Mitglieder in den Mittelpunkt mit dem Ziel „eine Organisation so zu gestalten, dass sie kontinuierlich ihre Fähigkeit erweitert, ihre Zukunft zu gestalten" (Senge, 1990, S. 3, 14, zit. in Wahren, 1996, S. 7). Die Stufe des Prozesslernens nach Argyris und Schön (1999) kann damit durch ein Denken in Systemen erweitert werden, womit die ganzheitliche Betrachtung der Organisation zum Ziel wird. Dieses systemische Denken hat eine integrierende Funktion und ist verschiedenen Dimensionen der Personal- und Organisationsentwicklung übergeordnet: Der Persönlichkeitsentwicklung der einzelnen Organisationsmitglieder ebenso wie dem kollektivem Lernen, den expliziten und impliziten Grundannahmen und deren Kommunikation sowie der gemeinsamen Vision bzw. Werten und Zielen der Organisation übergeordnet. Demnach sind eine Denkweise und eine Sprache zu entwickeln, mit der die Kräfte und Wechselbeziehungen, die das Verhalten des Systems steuern, zu begreifen und zu beschreiben sind. Auf diese Weise können typische Verhaltensmuster erkannt und besprechbar gemacht werden. Dies ermöglicht „Veränderungen in einer systemischen Betrachtungsweise im Unterschied zu monokausalen Ursache-Wirkungszusammenhängen anzugehen" (Seufert, 2013, S. 61). In diesem Sinne kommt auch der engen Verbindung von Personal- und Organisationsentwicklung eine grundlegende Rolle zu: Die Innovationsfähigkeit ist „untrennbar verbunden ... mit kompetenten Menschen und wandlungsfähigen Unternehmen. Personal-, Organisations- und Kompetenzentwicklung stehen damit in einem ganzheitlichen Innovationsmanagementsystem gleichberechtigt nebeneinander." (Haarich, Sparschuh, Zettel \& Hees, 2011, S. 448).

Der hier vorgestellte Ansatz zur Erhöhung der Lern- und Innovationsfähigkeit von Organisationen integriert diese systemische Denkweise und nimmt die Ebenen des Lernens von Individuen, Teams und Organisationen auf. Im Folgenden wird nun die Verzahnung von Personalentwicklung (Lernen von Individuen) und Organisationsentwicklung (Entwicklungsfähigkeit der Gesamtorganisation) aufgegriffen.

\section{Rahmenkonzept zur Steigerung der Lern- und Innovationsfähigkeit von Organisationen}

Die Lern- und Innovationsfähigkeit von Organisationen kann durch individuelle und organisationale Hindernisse beträchtlich negativ beeinflusst werden. Zur gezielten Steigerung der Lern- und Innovationsfähigkeit sind folglich organisationale Barrieren zu beseitigen und die Strategie, Strukturen und Kulturen so zu gestalten, dass Scheuklappen, Korsett sowie innovationsfeindliche Routinen (vgl. oben) aufgehoben werden können.

Als möglicher Ansatz wird hier eine stärkere Verzahnung von Personalentwicklung (PE) und Organisationsentwicklung (OE) vorgeschlagen. Dabei ergeben sich innerhalb des Rahmenkonzepts vier zentrale Handlungsfelder, die in der nachfolgenden Abbildung zunächst veranschaulicht sind: 


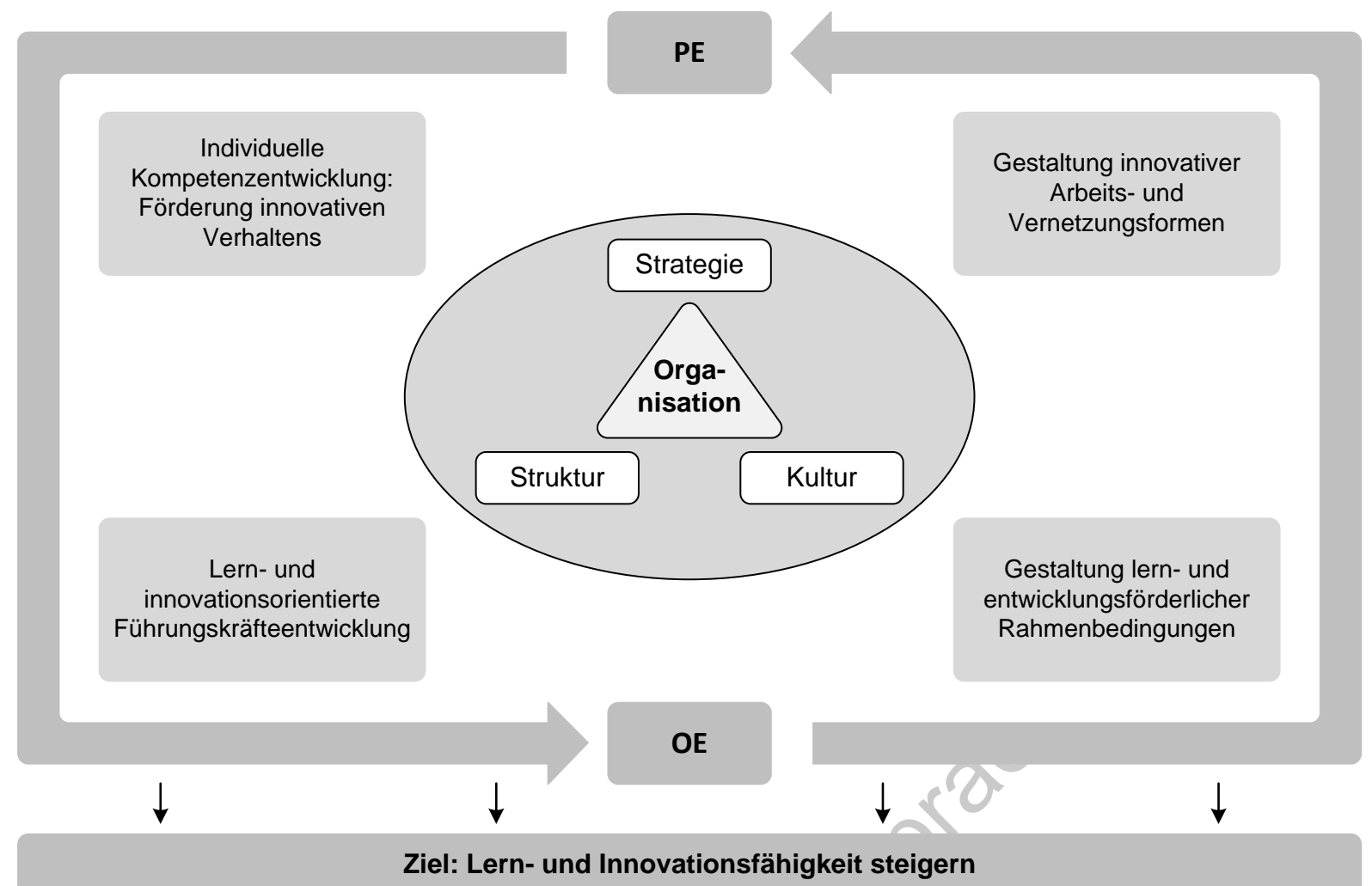

Abbildung 3: Rahmenkonzept - Konsequente Verzahnung von PE und OE

- Individuelle Kompetenzentwicklung: Schlüsselelemente innovativen Verhaltens von einzelnen Organisationsmitgliedern sind gezielt zu fördern, damit diese durch Lernprozesse entsprechende Kompetenzen entwickeln können. Zudem sind Determinanten innovativen Verhaltens zu beachten und bewusst zu gestalten, so dass es den Mitgliedern überhaupt möglich wird, sich lern- und innovationsorientiert zu verhalten.

- Lern- und innovationsorientierte Führungskräfteentwicklung: Gleichzeitig stellt PE, die auf Lernen und Innovationen gerichtet ist, auch eine Führungsaufgabe dar. Daher spielt die Befähigung von Führungskräften für die Förderung innovativen Verhaltens bei den Mitarbeitenden eine wichtige Rolle. Führungskräfte müssen in der Lage sein, lern- und innovationsförderliche Faktoren bewusst zu gestalten und über dafür notwendigen Kompetenzen verfügen. Das Bildungsmanagement nimmt dabei eine für die Führungskräfte unterstützende Funktion ein.

- Innovative Arbeits- und Vernetzungsformen: Um ein auf Innovationen ausgerichtetes Verhalten durch eine entsprechende Kompetenzentwicklung zu fördern, ist eine Verzahnung individuellen und organisationalen Wissens anzustreben. Damit einher gehen verschiedene Organisationslogiken für das Design von Kompetenzentwicklungsmassnahmen sowie die Ermöglichung „neuer“ Vernetzungsformen unter Mitarbeitenden und zwischen Mitarbeitenden und Kunden, die v.a. durch die neuen technologischen Entwicklungen begünstigt werden.

- Gestaltung lern- und entwicklungsförderlicher Rahmenbedingungen: Für den Abbau von Lern- und Innovationsbarrieren sind Kulturen und Strukturen mit entsprechenden Anreizsystemen und einer förderlichen Infrastruktur zu gestalten. Die systematische Erfassung solcher Faktoren zu einer Standortbestimmung kann durch Diagnoseinstrumente gelingen und auf dieser Basis einen Ausgangspunkt für Entwicklungsinitiativen darstellen.

Im folgenden Kapitel werden diese Handlungsfelder näher erläutert. 


\section{Zentrale Handlungsfelder zur Erhöhung der Lern- und Innovationsfähigkeit von Organisationen}

\subsection{Individuelle Kompetenzentwicklung: Förderung innovativen Verhaltens}

\subsubsection{Elemente innovativen Verhaltens}

Lernprozesse stellen die Grundlage für innovatives Verhalten der einzelnen Mitglieder dar; sie sind eine Voraussetzung für organisationales Lernen und damit für die Lern- und Innovationsfähigkeit von Organisationen. Daher ist es bedeutend, das Lernen und die Kompetenzentwicklung der Individuen zu fördern und mit entsprechenden Rahmenbedingungen zu ermöglichen. In diesem Verständnis und im Sinne einer verhaltenstheoretischen Perspektive deklariert Haller (2003) „Innovationsprobleme“ als „Verhaltensprobleme“ (S. 3). Innovatives Verhalten von Einzelnen gründet nach Haller (2003, S. 111154) auf vier Elementen, die im Hinblick auf die Förderung von Innovation zu berücksichtigen sind:

\begin{tabular}{|c|c|}
\hline Element & Beschreibung \\
\hline Sensibilität & $\begin{array}{l}\text { - Fähigkeit, sich anbahnende Veränderungen wahr- und aufzunehmen: „Je } \\
\text { sensibler das Unternehmen bzw. der Mensch ist, desto früher werden } \\
\text { Veränderungen unabhängig von ihrer Richtungsänderung wahrgenommen“ } \\
\text { (Haller, 2003, S. 111). }\end{array}$ \\
\hline Offenheit & $\begin{array}{l}\text { - Zugänglichkeit für wahrgenommene Veränderungen sowie damit verbunden die } \\
\text { vorurteilsfreie und bewusste Verarbeitung vielfältiger Informationen zu deren } \\
\text { Interpretation sowie das Zulassen von Konsequenzen auch gegen eigene } \\
\text { Erwartungen/ Vorstellungen. Mangelnde Offenheit führt dagegen zu einer } \\
\text { „optionenvermindernden Wahrnehmungsweise“ (Haller, 2003, S. 119). }\end{array}$ \\
\hline Wissen & $\begin{array}{l}\text { - Bildet die Grundlage des Aufbaus von Handlungsvermögen in } \\
\text { Innovationsprozessen: „Je mehr ein Mensch weiss, umso eher ist er in der Lage, } \\
\text { neue Ideen und neues Wissen wiederum zu generieren. Die vorhandene } \\
\text { Wissensbasis ist zudem Grundlage für das Verhalten gegenüber der Umwelt“ } \\
\text { (Haller, 2003, S. 130). } \\
\text { - Wissen entsteht aus der Vernetzung von Informationen durch den Austausch von } \\
\text { Informationen mit anderen Menschen oder aus Erfahrungen mit der Umwelt. }\end{array}$ \\
\hline Kreativität & $\begin{array}{l}\text { - Fähigkeit, Ideen zu generieren, die neu sind, d.h. ungewöhnliche bzw. originelle } \\
\text { Ideen, für die es noch keine Wertigkeit gibt }\end{array}$ \\
\hline
\end{tabular}

Tabelle 2: Elemente innovativen Verhaltens von Individuen (in Anlehnung an Haller, 2003, S. 111112)

Der Schlüsselfaktor Wissen ist für jeden Teil des Innovationsprozesses bedeutend. Im nächsten Abschnitt wird daher auf Wissensarten, im Speziellen das Arbeitsprozesswissen als Verbindung von Erfahrung und Theoriewissen sowie die Entwicklung von Kompetenzen eingegangen, die das erfolgreiche Handeln in zunehmend unvorhersehbaren und unsicheren Situationen ermöglichen. Anschließend wird auf Determinanten eingegangen, die innovatives Verhalten von Mitarbeitenden fördern oder auch hindern können.

\subsubsection{Wissensarten: Arbeitsprozesswissen und Kompetenzen}

Handlungskompetenzen beziehen sich nicht auf einmalige Verhaltensweisen, sondern auf innere Potenziale beziehungsweise Dispositionen für ein stabiles und regelmäßiges Handeln in bestimmten Situationen. Grundlegend ist somit die Unterscheidung von beobachtbaren Verhaltensweisen und zugrunde liegenden Handlungskompetenzen, die ein solches Verhalten ermöglichen.

Handlungskompetenzen werden damit als inneres Potenzial eines Menschen aus dem äußeren 
Verhalten interpretiert. Kompetenz bezeichnet somit das Potenzial eines Menschen, die sachlichen, sozialen und personalen Anforderungen innerhalb der Arbeitsbereiche bzw. Aufgaben eines Berufs zu bewältigen.

Berufliche Handlungskompetenz zeichnet sich auch dadurch aus, dass die Personen ein „Gespür“ bzw. eine Intuition entwickeln, um ohne langes Nachdenken in unsicheren bzw. unvorhergesehenen Situationen augenblicklich Lösungen zur Verfügung zu haben (vgl. Böhle, Pfeiffer \& Sevay-Tegethoff, 2004). In diesem Sinne kann von „tacit skills“ gesprochen werden, was die „Anwendung intuitiven Wissens als komplexe Verbindung von Kognition und Emotion“ (Schuchmann \& Seufert, 2013, S. 425, nach Arnold, 2005) meint. Ein solches intuitives Wissen ist vor allem durch Erfahrungslernen zu erwerben, was wiederum durch die reflexive Aufarbeitung von Handlungen und deren Auswirkungen möglich wird (Dehnbostel \& Pätzold, 2004, S. 27) . „Erfahrungslernen vollzieht sich stets als bewusstes, absichtliches und vor allem reflexives Lernen" (Lang \& Pätzold, 2004, S. 97, nach Dohmen, 2001, S. 34) und stellt eine Form des informellen Lernens dar. Lernen ist zunehmend in tagtägliche Arbeitsprozesse integriert, Arbeiten und Lernen verschmelzen immer mehr. In diesem Sinn wird „die intensive Verzahnung des Arbeitens und Lernens ... als ein wichtiger Schlüssel zur Innovationsfähigkeit (von Organisationen)" betrachtet (BMBF, 2007, S. 2). Damit verbunden sind dann auch neue Anforderungen an die Organisationen: „Es wird ... (davon) ausgegangen, dass die Zunahme wissensbasierter und wissensorientierter Arbeitsinhalte veränderte Arbeits- und Organisationsprozesse nach sich zieht, durch die direkt und vielfältig auf Lernen Einfluss genommen werden kann bzw. in denen Lernprozesse zur Wirkung kommen können“"(Salazar \& Peters, 2011, S. 127).

Diese Ausführungen verdeutlichen, dass formal organisiertes Lernen für eine auf innovatives Verhalten ausgerichtete Kompetenzentwicklung nicht ausreicht, vielmehr ist die Verzahnung informellen und formellen Lernens grundlegend (Kapitel 4.3.1 geht näher darauf ein). Der Begriff des Arbeitsprozesswissens vereint entsprechend Theoriewissen und praktische Erfahrung (vgl. nachfolgende Abbildung).

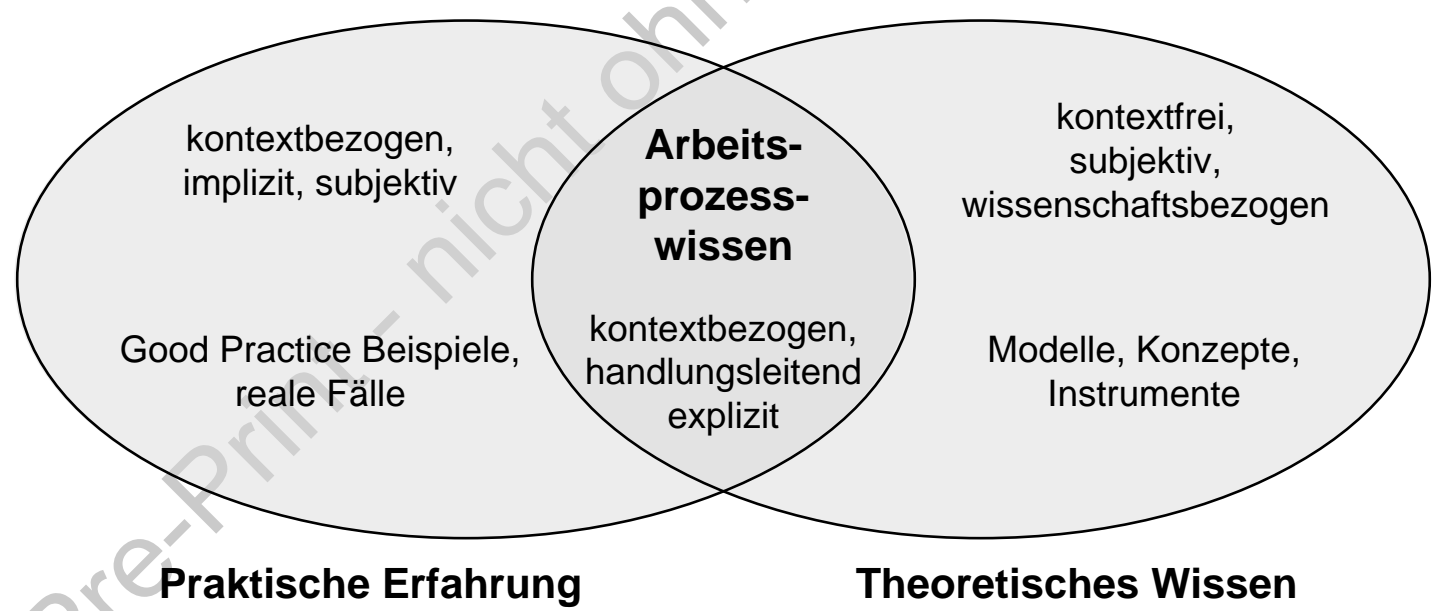

Abbildung 4: Arbeitsprozesswissen (in Anlehnung an Lang und Pätzold, 2004, S. 99, nach Fischer, 2002)

Lang und Pätzold (2004) definieren das Arbeitsprozesswissen nach Fischer (2000) wie folgt:

- „dasjenige Wissen, das im Arbeitsprozess unmittelbar benötigt wird (im Unterschied z.B. zu einem fachsystematisch strukturierten Wissen);

- es wird meist im Arbeitsprozess selbst erworben, z.B. durch Erfahrungslernen, schließt aber die Verwendung fachtheoretischer Kenntnisse explizit mit ein;

- es umfasst einen vollständigen Arbeitsprozess, im Sinne der Zielsetzung, Planung, Durchführung und Bewertung der eigenen Arbeit im Kontext betrieblicher Abläufe“. (S. 98) 
Es kennzeichnet sich durch „Personengebundenheit, ... Bezug zu situativen und konkreten Gegebenheiten und der Verbindung mit praktischem Handeln ... (Kontextwissen)“ (Lang \& Pätzold, 2004, S. 98). Zudem beinhaltet es Wissen über die Bewerkstelligung routinierter Handlungsabläufe und bekannter Aufgaben, geht aber auch darüber hinaus und beinhaltet ebenso Wissen darüber, dass sich an Routineabläufen plötzlich etwas ändern kann, worauf schnellstmöglich kompetent reagiert werden muss. Ausgangspunkt für die Entwicklung von Arbeitsprozesswissen sind - wie bereits oben angedeutet - „spezifische Problemsituationen, zu deren Bewältigung sich das Arbeitsprozesswissen auch bewähren muss" (Lang \& Pätzold, 2004, S. 101).

Bisher erfolgte der Erwerb von Arbeitsprozesswissen in informellen Lernprozessen, d.h. beiläufig. Dieser beiläufige Wissenserwerb wird jedoch aus verschiedenen Gründen (z.B. Notwendigkeit verkürzter Produkt- oder Innovationszyklen) mehr und mehr in Frage gestellt (Lang \& Pätzold, 2004, S. 98) und die Forderung nach gezielter Förderung kommt auf. So ist einerseits ein systematisches Erfahrungslernen zu ermöglichen, andererseits auch die Reflexion gemachter Erfahrungen. Darüber hinaus ist Erfahrungslernen durch strukturierte formelle Lernangebote zu ergänzen und zwar so, dass das (eher unstrukturierte) Erfahrungswissen mit Theoriewissen verbunden und damit strukturiert wird.

Mit diesen Ausführungen wird somit deutlich, dass berufliche Handlungskompetenz mehr ist als Wissen, zugleich auch mehr als ausführendes Tun. Es verbindet Reflexion und Aktion, d.h. Kennen, Können und Wollen bzw. Wissen, Fertigkeiten und Einstellungen. Damit Mitarbeitende innovatives Können (also Fertigkeiten) am Arbeitsplatz zeigen können, sind Wissen (insbesondere erfahrungsbasiertes Arbeitsprozesswissen) sowie entsprechende Einstellungen im Sinne von Grundhaltungen gegenüber innovativen Verhaltensweisen notwendig (s. Abschnitt 4.4.1. Sensibilität, Offenheit, etc.).

\subsubsection{Erfolgsfaktoren für innovatives Verhalten von Organisationsmitgliedern}

Innovatives Verhalten der einzelnen Organisationsmitglieder basiert auf vier Determinanten, denen für dessen Förderung Bedeutung zukommt (Haller, 2003, S. 192ff.). Es sind zum einen personale Faktoren, also im Individuum selbst verankert, zum anderen situative Faktoren, die in der Organisation als Umwelt zu verorten sind. Gleichzeitig kommt der Organisation bzw. den Führungskräften die Aufgabe zu, solche Rahmenbedingungen schaffen, die sich positiv auf die personalen Faktoren auswirken. Die nachfolgende Abbildung stellt die vier Determinanten und deren Beziehung zueinander dar.

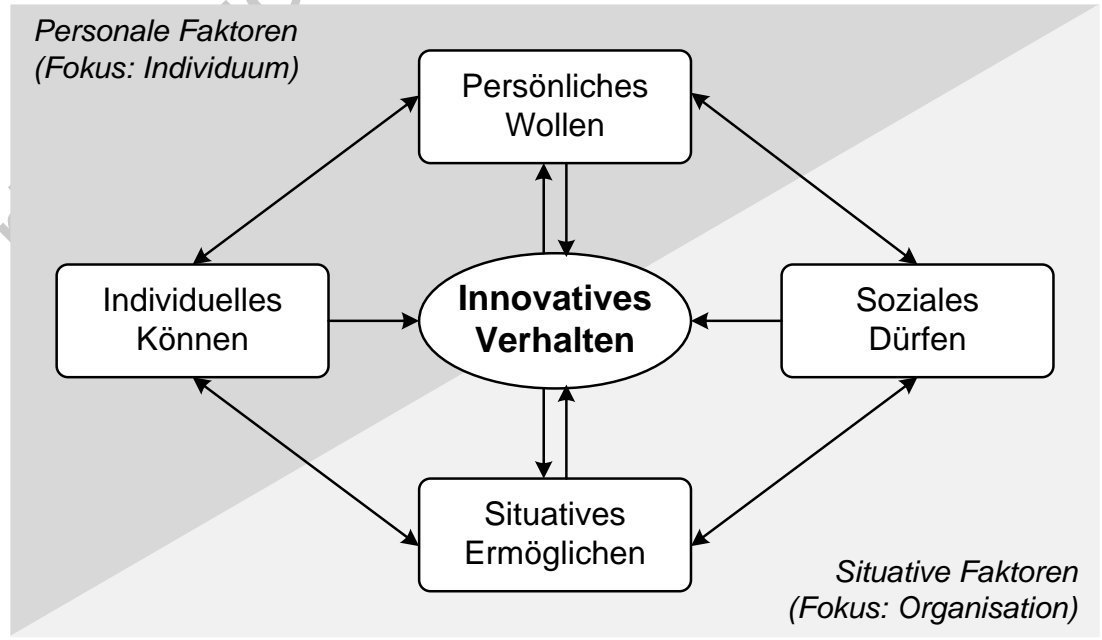

Abbildung 5: Determinanten innovativen Verhaltens (in Anlehnung an Haller, 2003, S. 193)

Wie die Abbildung aufzeigt, sind diese Determinanten nicht getrennt voneinander zu betrachten, sondern stets im Zusammenspiel. Positiv wirkende situative Faktoren reichen noch nicht aus, damit eine Person innovatives Verhalten zeigt. Ebenso müssen die personalen Faktoren für das Verhalten förderlich sein. Genauso wird sich innovatives Verhalten nicht zeigen, wenn bspw. persönliches 
Wollen nicht gegeben ist. Es wird deutlich, dass diese Determinanten mögliche Barrieren für innovatives Verhalten von Einzelnen darstellen, wenn sie sich negativ auf dieses auswirken. Im Folgenden wird näher auf die Determinanten sowie ihre Ausprägungen eingegangen.

Das situative Ermöglichen umfasst Voraussetzungen in der Umwelt, die geschaffen sein müssen, damit ein bestimmtes Verhalten stattfinden kann. Vor allem sind ein angemessener Zugang zu Informationen, aber auch strukturelle Rahmenbedingungen zu erwähnen. Mit dieser Forderung einher geht eine direkte Kommunikation für problemorientierten Informationsaustausch, was v.a. informeller Kommunikation bzw. informellem Austausch (auch zur Entwicklung von Arbeitsprozesswissen) große Bedeutung einräumt. Dies kann spontan und aus eigener Initiative des Einzelnen ausgehen: „die Befähigung zur Generierung und Adaption von Lösungen über interaktive Wissensgenerierung, Informationsselektion und Erfahrungsaustausch ist eine wichtige Voraussetzung für die Innovationsfähigkeit von Unternehmen“ (Haarich et al., 2011, S. 456). Darüber hinaus sind entsprechende Ressourcen und Kapazitäten wie z.B. Lernzeit für die Mitarbeitenden zu schaffen.

Das soziale Dürfen bezieht sich v.a. auf die Unternehmenskultur als Rahmenbedingung, die eine Erwünschtheit oder Unerwünschtheit von Verhalten implizieren, z.B. durch ein in der Organisation verankertes „Korsett“. Damit verbunden sind Erwartungen von Vorgesetzten oder Kollegen sowie die entsprechende Unterstützung, die von ihnen ausgeht. Es ist also eine innovationsfördernde Unternehmenskultur zu etablieren, die entsprechende Normen und Werte für innovatives Verhalten von Einzelnen beinhaltet. Diese räumen den Schlüsselfaktoren Sensibilität, Offenheit und Kreativität Platz ein, indem sie sie zulassen und aktivieren. Weiterhin ist die Internalisierung solcher Unternehmenswerte durch die Einzelnen eine Grundlage (Erpenbeck, 2004, S. 52; Haller, 2003, S. 293).

Das Individuelle Können meint die grundsätzlichen individuellen Fähigkeiten, ein bestimmtes Verhalten überhaupt zu zeigen. „Individuelles Können befähigt den Einzelnen, sich adäquat an Innovationsprozessen beteiligen zu können. Die Summe allen individuellen Könnens im Unternehmen beeinflusst wiederum die Qualität des Outputs und der Zusammenarbeit in Innovationsprozessen." (Haller, 2003, S. 337) Daher ist es wichtig, dass die Einzelnen ihre Kompetenzen (nicht nur Wissen!) kontinuierlich (weiter) entwickeln. Neben entsprechender Kompetenzen ist ein „höchstmöglicher Wertekonsens zwischen Unternehmen und Mitarbeitern" (Haller, 2003, S. 301) innovationsförderlich, d.h. die individuellen Werthaltungen der Mitarbeitenden und die kulturellen Unternehmenswerte sollten sich idealerweise weitestgehend decken, damit das Unternehmen erfolgreich agieren kann und was auch die Nichtimitierbarkeit fördert. Darüber hinaus kann innovatives Verhalten basierend auf individuellem Können gesteigert werden, indem Interaktion einen hohen Stellenwert im Unternehmen einnimmt, persönliche Interessen beim Einsatz von Mitarbeitenden berücksichtigt werden und Teamarbeit gefördert wird. (Haller, 2003, S. 310ff.)

Das Persönliche Wollen umfasst die Motivation und Bereitschaft einer Person, ein bestimmtes Verhalten zu zeigen. Einflussfaktoren sind Anreize, die individuelle Werthaltung sowie das Führungsverhalten des Vorgesetzten. Weniger geht es hierbei um die „aktive Motivation durch Dritte, sondern vielmehr (um) die Vermeidung von Demotivation bzw. von falsch gelenkter Aufmerksamkeit. Innovatives Verhalten lässt sich leicht ,destruieren'." (Haller, 2003, S. 341) Dieser Schlüsselfaktor fokussiert zwar die Person selbst, Demotivation könnte allerdings durch die Umwelt aufkommen.

Diese Ausführungen beschreiben einige Ansatzpunkte für die Förderung der individuellen Kompetenzentwicklung und damit des innovativen Verhaltens von einzelnen. Situative Einflussfaktoren auf innovatives Verhalten sind in der Strategie, in Strukturen und Kulturen Organisation verankert und sind lern- und innovationsförderlich zu gestalten. Personale Faktoren können durch die Organisation ebenso beeinflusst werden. In beiden Bereichen kommt gerade auch den Führungskräften eine bedeutende Rolle zu. Der nächste Abschnitt geht daher auf eine entsprechende lern- und innovationsorientierte Führungskompetenzen ein, die zu entwickeln sind. 


\subsection{Lern- und innovationsorientierte Führungskräfteentwicklung}

\subsubsection{Führungskompetenzen als Voraussetzung der Förderung innovativen Verhaltens}

In lern- und entwicklungsorientierten Unternehmen kommt Führungskräften bei der Unterstützung und Förderung des innovativen Verhaltens der Mitarbeitenden eine wichtige Rolle zu. Als „direkter Repräsentant" (Haller, 2003, S. 334) einer gelebten lern- und innovationsfördernden Unternehmenskultur sollten sie in ihrer Vorbildrolle in besonderem Masse Unternehmenswerte und -normen internalisieren. Führungskräfte sollten also neben den oben aufgeführten drei Kompetenzbereichen - Fach-, Sozial- und Selbstkompetenzen - ebenso über Führungskompetenzen verfügen, welche übergreifend zu verstehen sind. Haller (2003) beschreibt diese so: „Fähigkeiten, Ziele und Ressourcen priorisieren zu können und Transparenz zu wahren bzw. zu schaffen, auch in komplexen Situationen. Fähigkeit, Menschen zu begeistern und für gemeinsame Ziele einzunehmen, deren Potenziale zu erkennen und zu fördern“ (S. 297). Die nachfolgende Abbildung ordnet diese vier Kompetenzbereiche nach dem Grad ihrer Beeinflussbarkeit im Rahmen der Führungskräfteentwicklung sowie ihrem jeweiligen relativen Potenzial zur Förderung von Innovationen ein.

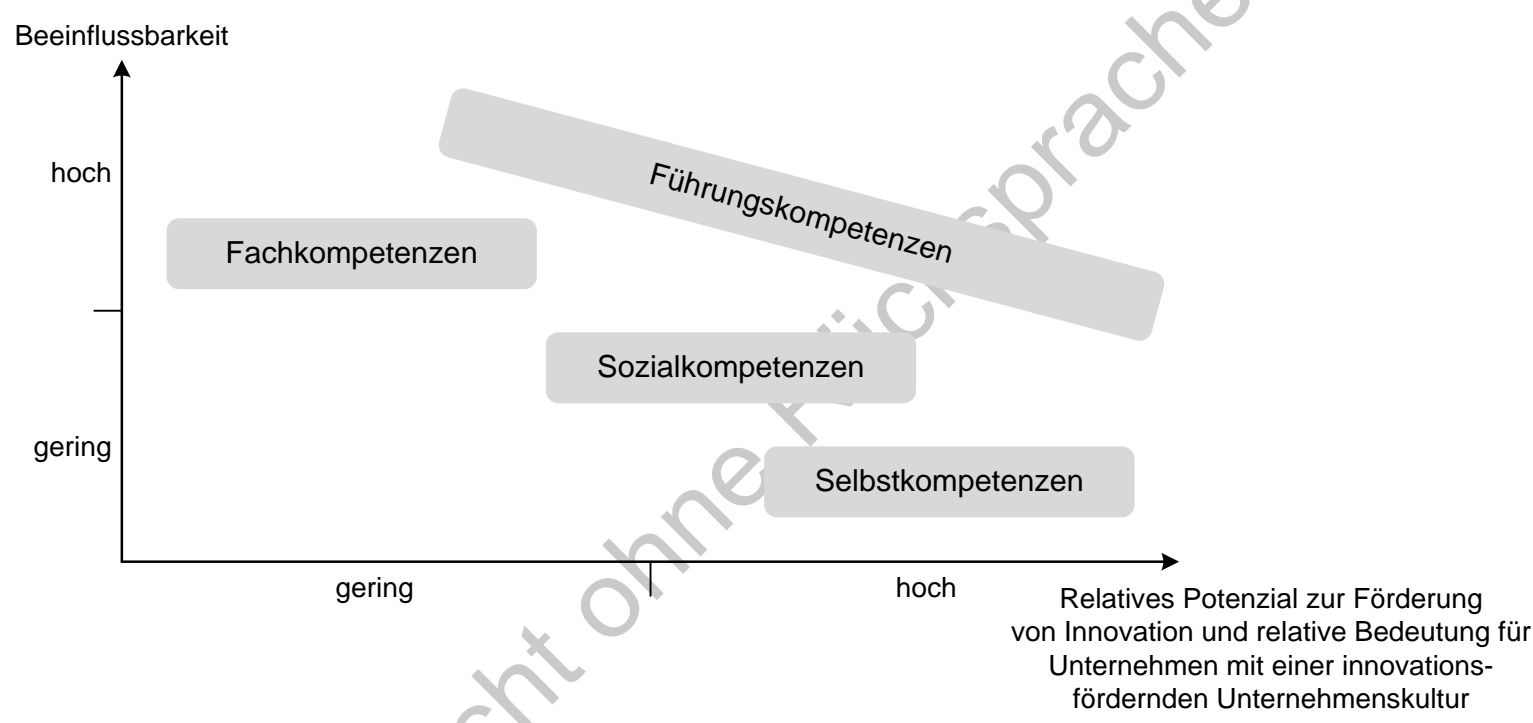

Abbildung 6: Kompetenzen und deren relative Bedeutung für Innovation sowie Beeinflussbarkeit (in Anlehnung an Haller, 2003, S. 298)

Sozial- und Persönlichkeitskompetenzen sind in besonderem Masse für Führungskräfte von Bedeutung, um Potenziale der Mitarbeitenden entsprechend fördern zu können. Oftmals werden in der Praxis allerdings Führungskräfte aufgrund von Fachkompetenzen oder kreativem Potenzial ausgewählt, ohne soziale Führungskompetenzen zu berücksichtigen. Jedoch steht nicht ihr eigenes Potenzial für innovatives Verhalten im Vordergrund, sondern dessen Förderung durch Führungskräfte bei den Mitarbeitenden - sie müssen also „bei anderen kreatives Potenzial entdecken und weiterentwickeln können. Die ideale Führungskraft ist nicht bestrebt, selbst als ,der beste Innovator oder Erfinder' zu gelten, sondern Mitarbeiter dazu anzuleiten, die ,besten Innovatoren' des Unternehmens zu werden“ (Haller, 2003, S. 334) und dafür braucht es mehr als Fachkompetenzen.

\begin{tabular}{|l|l|}
\hline $\begin{array}{l}\text { Elemente } \\
\text { innovationsförderlichen } \\
\text { Verhaltens }\end{array}$ & \multicolumn{1}{c|}{$\begin{array}{c}\text { Anforderungen an Führungskräfte, } \\
\text { die innovatives Verhalten bei Mitarbeitenden (MA) fördern } \\
\Downarrow\end{array}$} \\
\hline $\begin{array}{l}\text { Hohe Sensibilität } \\
\text { gegenüber } \\
\text { Veränderungen }\end{array}$ & $\begin{array}{l}\text { Ausprägung der Sozial-, Selbst- und Führungskompetenzen } \\
\text { umgehen }\end{array}$ \\
\hline
\end{tabular}




\begin{tabular}{|c|c|}
\hline & $\begin{array}{l}\text { - Veränderungen innerhalb und außerhalb der Organisation sowohl } \\
\text { mental als auch von ihrer Haltung mittragen, } \\
\text { - für MA einen Raum zum angstfreien und von Neugierde geprägten } \\
\text { Raum schaffen zum Umgang mit Veränderungen sowie Erneuerungen }\end{array}$ \\
\hline $\begin{array}{l}\text { Grosse Offenheit } \\
\text { gegenüber Neuem und } \\
\text { anderen Denkweisen }\end{array}$ & $\begin{array}{l}\text { - jede neue Anregung und Idee ernstnehmen und versuchen, die } \\
\text { positiven Aspekte darin zu erkennen, } \\
\text { - mit eigenen Motiven und Gefühlen umgehen können und sie anderen } \\
\text { MA mitteilen, wodurch diese angeregt werden, sich ebenso zu } \\
\text { verhalten, } \\
\text { - Fähigkeit zum ganzheitlichen, Systemzusammenhänge und } \\
\text { Vernetzungen berücksichtigenden Denken, um so multikausale und } \\
\text { mehrdimensionale Ursachenketten und Wirkungen des eigenen } \\
\text { Verhaltens zu erkennen und als ein Teil eines Systems zu begreifen, } \\
\text { das nur verständlich wird, wenn die notwendige mentale Offenheit } \\
\text { entscheidende Systemelemente nicht,ausblendet'. }\end{array}$ \\
\hline $\begin{array}{l}\text { Hoher Stellenwert von } \\
\text { Wissen und } \\
\text { Wissensträgern }\end{array}$ & $\begin{array}{l}\text { - MA als eigenständige Menschen behandeln, nicht als disponierbare } \\
\text { Ressource, } \\
\text { - Fähigkeit, sich mit MA schnell und direkt auszutauschen und die } \\
\text { fachliche sowie soziale Botschaft der Informationen erkennen } \\
\text { - Ideen anderer anerkennen und MA die Chance lassen, ihre Ideen und } \\
\text { Arbeitsergebnisse selbst vorzutragen, } \\
\text { - fachliche und überfachliche Weiterbildung der MA fördern und bei sich } \\
\text { selbst rechtzeitig Wissenslücken erkennen und offensiv damit umgehen. }\end{array}$ \\
\hline $\begin{array}{l}\text { Zulassen und Fördern } \\
\text { von Kreativität und } \\
\text { unkonventionellen } \\
\text { Problemlösungen }\end{array}$ & $\begin{array}{l}\text { - innovatives Verhalten der Mitarbeiter fördern als normaler und } \\
\text { selbstverständlicher Bestandteil des täglichen Handelns, } \\
\text { - dabei selbst eine hohe Lern- und Veränderungsbereitschaft zeigen und } \\
\text { Gewohntes in Frage stellen, } \\
\text { - anderen Mut machen zur Veränderung sowie Lust auf Innovation und } \\
\text { Leistung, } \\
\text { - Problemsensibilität und Entdecken von Widersprüchen bei MA fördern, } \\
\text { - einerseits kreative Produkte bei MA fordern und andererseits den } \\
\text { Verlauf kreativer Prozesse kennen. }\end{array}$ \\
\hline
\end{tabular}

Tabelle 3: Anforderungen an Führungskräfte, die innovatives Verhalten von MA fördern (in Anlehnung an Haller, 2003, S. 335)

\subsubsection{Führungskräfte als Personal- und Kompetenzentwickler}

Führungskräfte werden immer mehr dazu aufgefordert, ihre Rolle auch als Personal- und Kompetenzentwickler zu verstehen und „neben Management- und Sachaufgaben auch die Aufgabe einer professionellen Mitarbeiterentwicklung zu übernehmen" (Seufert, 2013, S. 262). Führungskräfte nehmen eine bedeutsame Vorbildrolle in der Organisation ein, sie sind „Agents of Change“ (Bass \& Stogdill, 1990, S. 18). Ihnen kommt damit auch eine Multiplikatoren-Rolle für Lernen und innovatives Verhalten ihrer Mitarbeitenden zu. Gleichzeitig sind sie „zentrale Kulturträger“ und können Veränderungen der lern- und innovationsförderlichen Unternehmenskultur begünstigen oder verhindern (Seufert, 2013, S. 263). „Oft sind Führungskräfte ... Mitverursacher für den ,innovationsfeindlichen' Zustand im Unternehmen“ (Haller, 2003, S. 294). Sie können die Unternehmenskultur grundlegend mitbeeinflussen. Entsprechend sind sie zu sensibilisieren und ein Bewusstsein zu schaffen, welche Möglichkeiten der Kulturgestaltung ihnen zukommen.

Gerade die mangelnde Unterstützung durch Führungskräfte führt in der Unternehmenspraxis oft zu großen Barrieren für eine lern- und entwicklungsförderliche Kultur. Die Frage nach einer geteilten Verantwortung von Vorgesetzten, Mitarbeitenden und Personal- bzw. Bildungsverantwortlichen ist 
ebenso in der Human Resource Development Forschung ein zentrales Thema (vgl. u.a. Manning, 2002). Gleichzeitig kommen neue Steuerungslogiken in Organisationen auf: „Nicht die Umsetzung von Vorgaben, sondern Selbstorganisation, Verständigung und Verantwortung sind wichtige organisationale Leitprinzipien. Hinzu kommt das Ausschöpfen der Potenziale." (Seufert, 2013, S. 317) Einerseits geht es dabei um die Sicherung von „High Potentials“, also Talenten, andererseits auch um die Förderung von „B-Playern“. Entgegen der kritischen Annahme mancher, dass die Förderung des individuellen Lernens dazu führe, dass sich Mitarbeitende weiterentwickeln und dann das Unternehmen verlassen würden, ist gerade das Gegenteil der Fall: Eine ausbleibende Unterstützung und ausbleibende Ermutigung zur individuellen Kompetenzentwicklung führt dazu, indem dies Unzufriedenheit bei den Mitarbeitenden zur Folge hat (Hart, 2013, S. 11). Mit den letzten Ausführungen wird die Frage nach der Rollenausgestaltung von Führungskräften auch aus einer ökonomischen Nutzenperspektive beleuchtet: Einige Studien bestätigen, dass Mitarbeitende Organisationen oft aus diesem Grund verlassen und nicht aus finanziellen Gründen (vgl. Gibb, 2003, S. 282). Zu betonen ist darüber hinaus der demografische Wandel und die damit verbundene Herausforderung eines erfolgreichen Talent Managements.

Eine solche geforderte Selbststeuerung und Innovationsorientierung der Mitarbeitenden kann durch einen passenden Führungsansatz gefördert werden. Während ein transaktionaler Führungsstil eher zu Anpassungslernen ermutigt, strebt ein transformationaler Stil an, dass Mitarbeitende sich für höhere, nämlich intrinsische Ziele einsetzen, die über ihre eigenen Interessen hinausgehen.. Indem der Sinn des Handelns und gemeinsame Ziele besonders verdeutlicht werden, wird das Verhalten bzw. Bewusstsein der Mitarbeitenden auf eine höhere Stufe gehoben. Eine Führungskraft mit einem solchen Verhalten ist imstande Werte zu vermitteln, Loyalität aufzubauen, kontinuierliche Verbesserung voranzutreiben, inspirierend, visionär zu agieren und eingefahrene Denkmuster zu überprüfen und aufzubrechen (Bruch, Krummacher \& Vogel, 2012, S. 111). Ergebnisse einer Studie von Geyer \& Steyrer (1998) bestätigten, dass ein solches Führungsverhalten für die Organisation langfristig erfolgsversprechend zu sein scheint.

Mit der Forderung nach Selbststeuerung der Kompetenzentwicklung von Mitarbeitenden einher geht auch die Forderung nach Auflösung hierarchischer Unternehmensstrukturen (Seibold, 1995, S. 230). Das umfasst bspw. das Denken in Projektteams, die sich nach Kompetenzen sowie individuellen Interessen zusammensetzen können. Die Unterstützung der Führungskräfte als eine neue Aufgabe des Bildungsmanagements in Unternehmen konkret aufzunehmen, ist eine zentrale Herausforderung. Vier Handlungsbereiche skizzieren die Möglichkeiten, die sich aus der Kombination von didaktischer und managementorientierter Abstimmung sowie Rahmen- und Interaktionsgestaltung von Führungskräften ergeben:

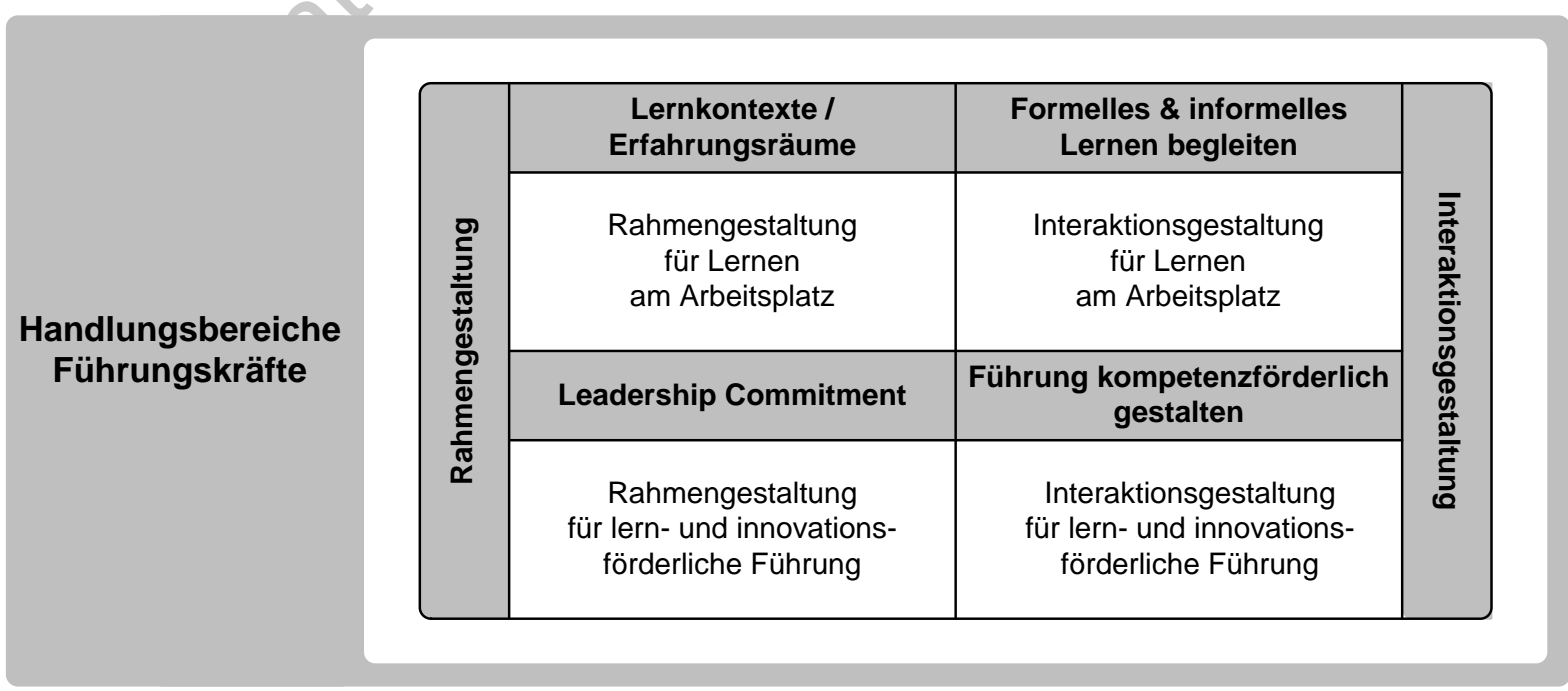


Abbildung 7: Handlungsbereiche von Führungskräften in ihrer lern- und entwicklungsfördernden Rolle (Seufert, 2013, S. 328)

Aufgabe des Bildungsmanagements in einem Unternehmen kann sich dabei in der Führungskräfteentwicklung darauf ausrichten, ihre Führungskräfte auf ihre veränderte Rolle und die konkreten Handlungsbereiche vorzubereiten und in ihrer täglichen Führungspraxis zu unterstützen.

\subsection{Gestaltung innovativer Arbeits- und Vernetzungsformen}

\subsubsection{Kompetenzentwicklung: Verzahnung von formellem und informellen Lernen}

Eine Kompetenzentwicklung, die auf innovatives Verhalten ausgerichtet ist, impliziert mehr als nur formal organisierte Kompetenzentwicklung. Auf der Grundlage der beiden Dimensionen: "formal organisiertes Lernen - informelles Lernen“ und „individuelles Lernen - organisationales Lernen“ können nach Seufert (2012) folgende Logiken zur Organisation von Lernprozessen unterschieden werden (vgl. nachfolgende Abbildung):

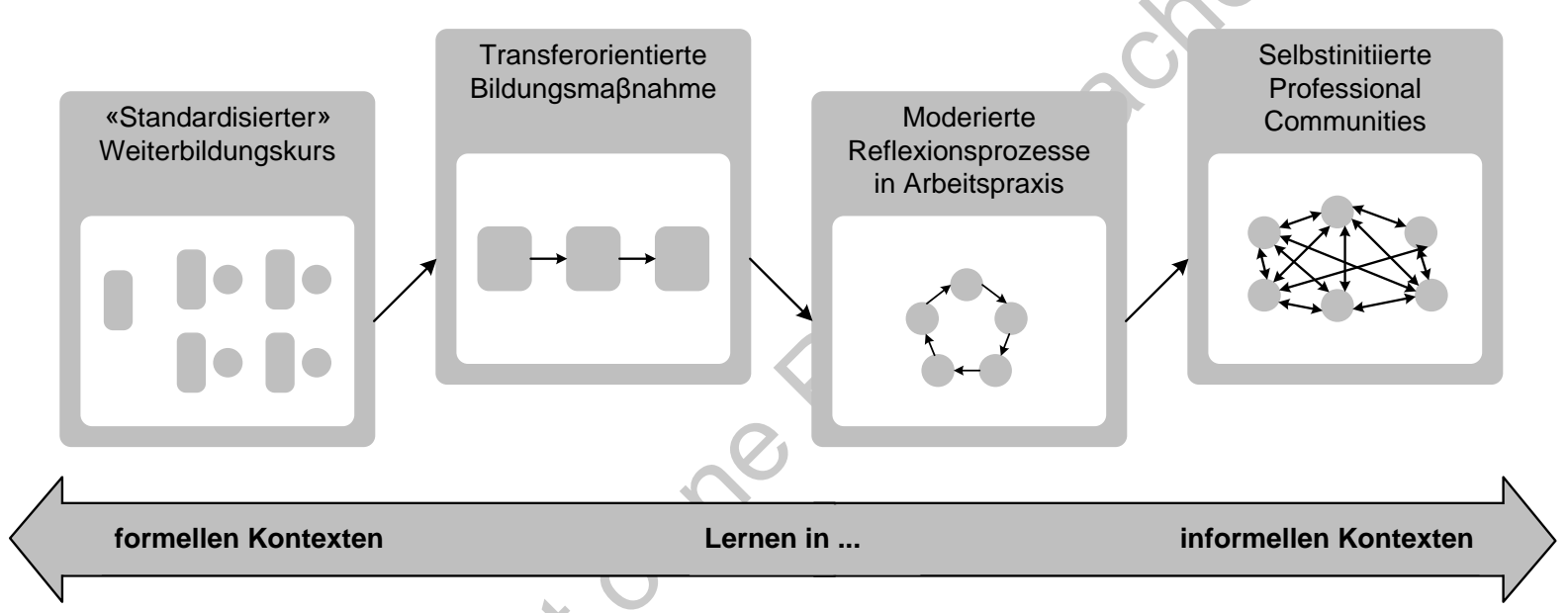

Abbildung 8: Organisationslogiken zur Gestaltung von Lernen (Seufert, 2012, S. 37)

Die nachfolgende Tabelle zeigt die drei unterschiedlichen Organisationslogiken für das Design von Bildungsmaßnahmen, um insbesondere Lernprozesse in informellen Kontexten zu integrieren (Seufert, 2011, S. 303, in Anlehnung an Poell et al., 2000, S. 25):

\begin{tabular}{|c|c|c|c|}
\hline Art & Ziele & Individuelles Lernen & $\begin{array}{l}\text { Organisationales } \\
\text { Lernen }\end{array}$ \\
\hline $\begin{array}{l}\text { "Extended } \\
\text { Training“ } \\
\text { Informelles } \\
\text { Lernen in Vor-I } \\
\text { Nachbereitung } \\
\text { integrieren }\end{array}$ & $\begin{array}{l}\text { Organisation eines } \\
\text { Lernprozesses (statt } \\
\text { punktuelle Events); } \\
\text { z. B. Vorbereitungs-, } \\
\text { Präsenz- und Nach- } \\
\text { bereitungsphase }\end{array}$ & $\begin{array}{l}\text { - Mithilfe informeller } \\
\text { Lernangebote den } \\
\text { individuellen } \\
\text { Transfererfolg } \\
\text { erhöhen, } \\
\text { - Angepasste } \\
\text { Arbeitsprozesse } \\
\text { bewirken. }\end{array}$ & $\begin{array}{l}\text { Schwerpunkt „Single } \\
\text { Loop Learning“: } \\
\text { - Effektive Adaption der } \\
\text { Organisation an } \\
\text { vorgegebene Ziele } \\
\text { und Normen, } \\
\text { - Anpassungslernen. }\end{array}$ \\
\hline $\begin{array}{l}\text { Moderiertes } \\
\text { Workplace } \\
\text { Learning } \\
\text { Reflexion am } \\
\text { Arbeitsplatz } \\
\text { unterstützen }\end{array}$ & $\begin{array}{l}\text { Angeleitete Reflexion } \\
\text { am Arbeitsplatz im } \\
\text { informellen Rahmen } \\
\text { eines konzipierten } \\
\text { Lernangebotes }\end{array}$ & $\begin{array}{l}\text { - Unterstützung von } \\
\text { Lernprozessen am } \\
\text { Arbeitsplatz, } \\
\text { - Problemlösung in } \\
\text { Teams und Auslösen } \\
\text { von (moderierten) } \\
\text { Reflexionsprozessen } \\
\end{array}$ & $\begin{array}{l}\text { Schwerpunkt „Double } \\
\text { Loop Learning“: } \\
\text { - Hinterfragung und ggf. } \\
\text { Restrukturierung der } \\
\text { existierenden } \\
\text { Handlungstheorie, } \\
\text { - Veränderungslernen. }\end{array}$ \\
\hline
\end{tabular}




\begin{tabular}{|c|c|c|c|}
\hline $\begin{array}{l}\text { (innovations- } \\
\text { orientiertes) } \\
\text { Lernen in } \\
\text { sozialen } \\
\text { Gemeinschaften }\end{array}$ & $\begin{array}{l}\text { Selbstorganisierte } \\
\text { Reflexion über } \\
\text { Innovationen innerhalb } \\
\text { einer Profession, } \\
\text { z. B. neue Arbeits- } \\
\text { methoden oder } \\
\text { Prozesse. }\end{array}$ & $\begin{array}{l}\text { - Eigeninitiierte } \\
\text { Reflexionsprozesse, } \\
\text { - Steigerung der } \\
\text { individuellen } \\
\text { Lernfähigkeit, } \\
\text { unterstützt durch } \\
\text { organisatorische/ } \\
\text { kulturelle Rahmen- } \\
\text { bedingungen. }\end{array}$ & $\begin{array}{l}\text { Schwerpunkt „Deutero } \\
\text { Learning“: } \\
\text { - Einsichten über die in } \\
\text { der Organisation } \\
\text { ablaufenden Lern- } \\
\text { prozesse selbst, } \\
\text { - Prozesslernen und } \\
\text { Steigerung der } \\
\text { Lernfähigkeit des } \\
\text { Unternehmens. }\end{array}$ \\
\hline
\end{tabular}

Tabelle 4: Organisationslogiken für das Design von Bildungsmaßnahmen in der betrieblichen Bildung

Insbesondere die letztgenannte Organisationslogik für das Design von Lernen in sozialen Arbeitsgruppen, Netzwerken und Communities bezieht sich darauf, Freiräume und die Selbstorganisation für das Entstehen von Innovationen zu unterstützen. Die Mitarbeitenden reflektieren in hoher Eigeninitiative selbst über Innovationspotenziale in ihrem Arbeitsbereich. Das heißt, sie entwickeln eigene Innovationsstrategien, um zu erkunden, inwieweit neuere Entwicklungen in ihrem Arbeitskontext relevant sind und inwiefern diese erfolgreich eingesetzt werden können. Häufig erhalten Mitarbeitende neue Impulse und Trends aus der Profession, der sie sich zugehörig fühlen. Die Orientierung an Entwicklungen in einer Profession ist daher für die Mitarbeitenden sehr wichtig, um potenzielle Innovationen für die eigene Arbeitspraxis zu reflektieren. Die Rolle des Bildungsmanagements besteht dann v.a. darin, lernförderliche Rahmenbedingungen zu schaffen, um sich - insbesondere auch extern - in professionellen Gemeinschaften auszutauschen. Ein Aspekt dabei ist sicherlich, Zugänge für informelles Lernen über vielfältige Formen, wie z.B. Blogs für „Trendwatching“, soziale Netzwerke etc., zu ermöglichen. Die Unterstützung der selbstorganisierten Reflexion steht im Einklang mit der Ebene des Deutero-Learnings, da das "Lernen lernen“ einen zentralen Stellenwert einnimmt, um schließlich die Lernfähigkeit einer Organisation insgesamt zu erhöhen. Letztendlich beinhaltet dieser Ansatz eine Renaissance des Community-Konzepts. Die Idee der praxisbezogenen Gemeinschaft von Personen (Community of Practice nach Wenger, 1998), die informell miteinander verbunden sind, ähnlichen Aufgaben gegenüberstehen und durch einen selbstorganisierten Austausch die Praxis in dieser Gemeinschaft prägen, ist grundsätzlich nicht neu. Neu scheint mit dem Aufkommen von Social Media jedoch zu sein, dass der Fokus weniger auf der Gemeinschaft, sondern vielmehr auf dem Individuum und dessen Beitrag für eine Gemeinschaft liegt (eher "me-centric" statt „we-centric").

Für die Unterstützung der einzelnen Teammitglieder bedeutet das, ihnen zu helfen, neue Kompetenzen für den Umgang mit einer solchen sozialen Arbeitsumgebung und digital vernetzten Mitarbeitenden zu entwickeln (um z.B. „oversharing“ zu vermeiden). Diese beziehen sich vor allem auf persönliches Wissensmanagement (wie kann ein Netzwerk aufgebaut werden, auf einer täglichen Basis Informationsquellen ausgemacht, wie diese sinnvoll genutzt und angemessen geteilt werden) sowie kollaboratives Arbeiten und Lernen in einem digital vernetzten Team.

\subsection{2 "Neue“ Vernetzungsformen zwischen Mitarbeitenden, Kunden und Partnern}

Das Social Web hat bereits die Art des Lernens von Individuen durch konstante Wissens- und Informationsflüsse verändert. In ähnlicher Weise wird sich das Lernen von Teammitgliedern innerhalb der Unternehmen durch Social Tools verändern, die im Unternehmen ihren Einsatz finden. Koch, Richter und Stocker (2012, S. 33) konnten bei einer Analyse von Fallstudien, die die Einführung von Social Software in 23 Unternehmen in Deutschland, Österreich und der Schweiz untersuchte, 6 Zielkategorien ausmachen, die mit Social Software adressiert werden: 


\begin{tabular}{|c|c|}
\hline Effiziente, $\quad$ zielorientierte & - Offenere Kommunikationskanäle \\
\hline Kommunikation und & - Direktere Mitarbeiter-zu-Mitarbeiter-Kommunikation \\
\hline $\begin{array}{ll}\text { Vermeidung } & \text { von } \\
\text { Informationsüberflutung } & \end{array}$ & $\begin{array}{l}\text { - Vermeidung bzw. bessere Bewältigung von Informationsüberflutung } \\
\text { - Verminderung des E-Mail-Aufkommens }\end{array}$ \\
\hline $\begin{array}{l}\text { Effizienter } \\
\text { Wissenstransfer }\end{array}$ & $\begin{array}{l}\text { - Vermeidung von Wissenssilos } \\
\text { - Verbesserung des innerbetrieblichen Wissenstransfers } \\
\text { - Besserer Zugang zu Best Practices }\end{array}$ \\
\hline $\begin{array}{l}\text { Partizipation der Mitarbeiter } \\
\text { und Schaffung einer offenen } \\
\text { Unternehmenskultur }\end{array}$ & $\begin{array}{l}\text { - Nachhaltige Einbeziehung der Mitarbeitenden } \\
\text { - Reduzierte Anonymität der Mitarbeitenden } \\
\text { - Entwicklung eines kreativen Klimas, in das sich die Mitarbeitenden } \\
\text { einbringen können und das auch gerne tun }\end{array}$ \\
\hline $\begin{array}{l}\text { Aufbau } \\
\text { Expertennetzwerken }\end{array}$ & $\begin{array}{l}\text { - Verbesserung der Expertenidentifikation und Mitarbeitervernetzung } \\
\text { - Mitarbeitende mit ähnlichem Kontext tauschen sich in Experten- } \\
\text { Communities aus } \\
\text { - Die Weisheit der Masse wird genutzt }\end{array}$ \\
\hline $\begin{array}{l}\text { Gesteigerte Awareness und } \\
\text { Transparenz }\end{array}$ & $\begin{array}{l}\text { - Bessere Sichtbarkeit der Aufgaben und Kompetenzen } \\
\text { - Mehr Transparenz über Entscheidungen und Prozesse } \\
\text { - „Serendipity“: Spürsinn, Zufallsfunde }\end{array}$ \\
\hline $\begin{array}{l}\text { Gesteigertes } \\
\text { Innovationspotenzial } \\
\text { Zukunftsfähigkeit }\end{array}$ & $\begin{array}{l}\text { - Innovationen können schneller aufgenommen werden } \\
\text { - Neue Systeme ermöglichen zusätzliche Flexibilität und die } \\
\text { Zukunftsfähigkeit des Unternehmens } \\
\text { - Die Nachhaltigkeit wird nach aussen auch für die junge Generation } \\
\text { gezeigt }\end{array}$ \\
\hline
\end{tabular}

Tabelle 5: Ziele der Einführung von Social Software in Unternehmen (in Anlehnung an Koch et al., 2012, S. 33)

Diese Punkte zeigen, dass Enterprise 2.0 eine interne sowie eine externe Dimension bezogen auf das Unternehmen haben kann (Stobbe, 2010, S. 4):

- Innenperspektive: hier geht es um die Nutzung von Web 2.0-Tools im Unternehmen mit dem Ziel, Prozesse zu verbessern, Zusammenarbeit zu fördern, Wissen und Informationen auszutauschen u.a.

- Aussenperspektive: dabei steht die Nutzung der Tools nach außen im Mittelpunkt, z.B. zur Kommunikation mit Kunden, Marketing- und Reputationszwecken, Zusammenarbeit mit Kunden, Zuliefern, Experten z.B. zur Produktentwicklung u.a. 


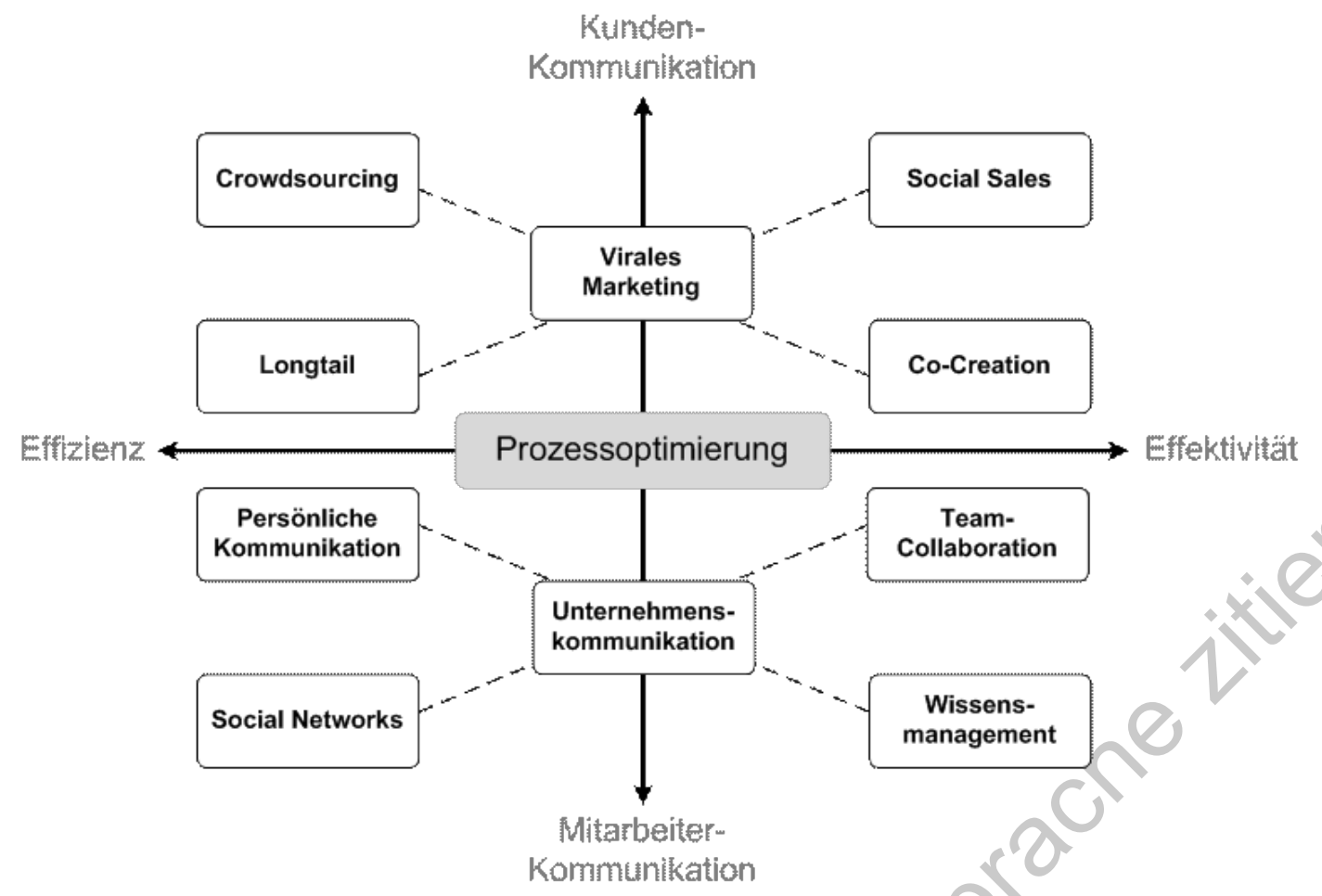

Abbildung 9: Vernetzung von Mitarbeiter- und Kundenkommunikation (nach Göhring, 2008, S. 165166)

Im Sinne der Förderung der Innovationsfähigkeit einer Organisation durch neue Arbeits- und Vernetzungsformen müssen beide hier aufgezeigten Dimensionen zusammen gedacht und zusammen behandelt werden. Die interne Vernetzung und Förderung des Dialogs von Mitarbeitenden (beispielsweise von Mitarbeitenden in bestimmten Fachgruppen) einerseits und die Vernetzung nach außen und Förderung des Dialogs mit Partnern und Kunden.

\subsection{Gestaltung lern- und innovationsförderlicher Rahmenbedingungen}

\subsubsection{Rahmenbedingungen: Anreizsysteme und Infrastrukturen gestalten}

Die Gestaltung von Strukturen und Kulturen in Organisationen bezieht sich auf das Ziel, lern- und innovationsförderliche Rahmenbedingungen für die individuelle Kompetenzentwicklung sowie für organisationales Lernen zu schaffen. Eine Organisation erscheint dabei im Allgemeinen nicht homogen, sondern vereint unterschiedliche Strukturen und Kulturen. Dabei beziehen sich die strukturellen Faktoren primär auf die Analyse und die Gestaltung von Aufgaben-, Entscheidungs-, Verantwortungs-, Budget- und Kommunikationsstrukturen. Demgegenüber erfassen die kulturellen Faktoren in erster Linie Einstellungen, Macht- und Vertrauensgrade, Werte, aber auch die impliziten und informellen Verhaltensweisen der Personen in einem sozialen System. Strukturelle Faktoren beziehen sich häufig auf die strukturellen Rahmenbedingungen, wie beispielsweise technologische Infrastrukturen und Ressourcen, die den Mitarbeitenden zur Kommunikation, Wissensmanagement, Lernprozesse, etc. zur Verfügung gestellt werden. Anreizsysteme als Rahmenbedingung in einer Organisation, um beispielsweise Wissen zu teilen, beziehen sich neben strukturellen Voraussetzungen, wie verfügbare Zeit, insbesondere auch auf kulturelle Aspekte (z.B. Transparenz, Ansehen und Reputation durch Bereitstellung von Wissen). Wilkesmann (1999, S. 199) schlägt vor, dass die Anreize für das Problemlösungslernen, das er auch als kollektives Innovationslernen bezeichnet, für die Organisationsmitglieder "diffus“ bleiben sollten. Ansonsten besteht die Gefahr, dass Innovationsroutinen erzeugt werden. 
In der Literatur existieren mittlerweile zahlreiche Ansätze, auch aus unterschiedlichen

Forschungssträngen, um relevante Faktoren zu definieren, welche eine hohe Relevanz als lern- und innovationsförderliche Rahmenbedingungen aufweisen:

\begin{tabular}{|l|l|}
\hline Operationalisierung des Konzepts der lernenden Organisation \\
\hline $\begin{array}{l}\text { Checklist of the } \\
\text { Learning Organisation } \\
\text { nach Pedler, Burgoyne \& } \\
\text { Boydell, 1991 }\end{array}$ & $\begin{array}{l}\text { Der Fragebogen zum Lernklima bezieht zehn Dimensionen von Lernen } \\
\text { ein: Umweltbedingungen, Lernressourcen, Ermutigung zu Lernen, } \\
\text { Kommunikation, Honorierung von Lernen, Konformität, Werte basierend } \\
\text { auf Idealen, praktische Hilfestellung, Verfügbarkeit von Support, } \\
\text { Standards. }\end{array}$ \\
\hline $\begin{array}{l}\text { Dimensions of the } \\
\text { Learning Organization } \\
\text { Questionnaire (DLOQ) } \\
\text { nach Yang, Watkins \& } \\
\text { Marsick, 2004 }\end{array}$ & $\begin{array}{l}\text { Dieses Instrument beinhaltet sieben Dimensionen zur lernenden } \\
\text { Organisation und zwei zur organisationalen Performanz: Kontinuierliche } \\
\text { Lernmöglichkeiten schaffen, Nachfragen und Dialog fördern, Ermutigung } \\
\text { zu Kollaboration und Teamlernen, eine gemeinsame Vision bei } \\
\text { Mitarbeitenden erreichen, Verbindung des Unternehmens mit seiner } \\
\text { Umwelt, System etablieren zur Erfassung und Teilen von Gelerntem, } \\
\text { strategische Führung für Lernen einrichten. }\end{array}$ \\
\hline
\end{tabular}

Kompetenzentwicklung und lernförderliche Rahmenbedingungen

\begin{tabular}{l|l} 
Lernkulturanalyse & Lernkultur zielt nach den Autoren auf „Kompetenzentwicklung,
\end{tabular}

nach Sonntag, Schaper Steigerung von Flexibilität und Innovationsfähigkeit im Unternehmen“"

\& Friebe, 2007

(Sonntag et al., 2007, S. 107) ab. Sie umfasst die Gesamtheit derjenigen

Faktoren, die das Lernen von Individuen in einer Organisation

beeinflussen. Diese können normative Aspekte, wie lernbezogene

Erwartungen umfassen sowie operative Aspekte, wie bspw.

Unterstützungsangebote, Anreizstrukturen und etablierte Lernformen

und -methoden. Dimensionen: Lernen als Teil der

Unternehmensphilosophie, organisationale Rahmenbedingungen für

Lernen, Aspekte der Personalentwicklung, Formalisierung von

Kompetenzentwicklung, Lernatmosphäre Unterstützung der Kollegen,

Lernorientierte Führung.

Lernkulturanalyse

Instrument zur Erhebung der Lernkultur als Ausgangspunkt für

nach Seufert,

Hasanbegovic \& Euler,

2007

Maßnahmen zur Organisationsentwicklung (Seufert et al., 2007, S. 19)

mit den Dimensionen: Mitarbeitende befähigen: wie und in welchem

Umfang wird bislang eigenverantwortliches Lernen gefördert und

gefordert, Führungskräfte einbinden, Lernen ermöglichen: welche

organisatorischen Rahmenbedingungen fördern (informelles) Lernen,

Lernen vielfältig gestalten: welche Formen formellen und informellen

Lernens sind bereits etabliert/akzeptiert?, Lernen einen Wert zuweisen:

wie werden Lernaktivitäten evaluiert und wie wird der Wert von Lernen

aufgezeigt und kommuniziert.

Innovationsförderliches Organisationsklima

Creative Climate

Questionnaire (CCQ)

nach Ekvall, 1996

Innovation Climate

Questionnaire

nach Kauffeld, Jonas,

Grote, Frey \& Frieling,

2004
Das Instrument fokussiert folgende Dimensionen: Herausforderung,

Handlungsfreiräume, Ideenunterstützung, Vertrauen/Offenheit,

Dynamik/Lebendigkeit, Verspieltheit/Humor, Debatten,

Risikobereitschaft, Konflikte, Ideenzeit

Messung des Innovationsklimas in Organisationen. Basierend auf einer

Faktorenanalyse wurden vier Dimensionen identifiziert:

Aktivierungsorientierte Führung, Unterstützung des Vorgesetzten bei

Problemlösungen und Entwicklung von Innovationen, kontinuierliche

Reflexion, wie die Organisation systematisch Schwächen/Mängel

identifiziert und Verbesserungen entwickelt, konsequente

Implementierungen, professionelle Dokumentation, Messung des Grades

schriftlichen Festhaltens von innovationsrelevanten Fakten. 
Tabelle 6: Überblick über ausgewählte Diagnoseinstrumente zur Analyse von lern- und innovationsförderlichen Rahmenbedingungen

Da viele Faktoren und auch ungeplante Ereignisse in kulturelle Veränderungsprozesse hineinspielen, muss davon ausgegangen werden, dass solche Veränderungsprozesse nur bis zu einem gewissen Grad gestaltbar sind. Dennoch kann die Gestaltung von Veränderungsprozessen - unter den geschilderten Einschränkungen - geplant und können Ziele gesteckt werden, auch wenn die Entwicklung nicht prognostizierbar ist und daher die Pläne oft nicht über längere Zeit aufrechterhalten werden können (Müller-Stewens \& Lechner, 2005, S. 412).

Zusammenfassend ist festzuhalten, dass das Prinzip der Selbstorganisation zur Erhöhung der Innovationsfähigkeit als zentraler Schlüsselfaktor gesehen wird und in verschiedener Hinsicht Anforderungen an Unternehmen und Organisationen stellt. In vielen Bereichen behindern derzeit starre Strukturen und Vorgaben sowie kulturelle Barrieren die Umsetzung. Eine neue Kultur des selbstgesteuerten Lernens steckt meist noch in den Anfängen (in Anlehnung an Dietrich, 2001, S. 314). So kommt Meiser (2001) zu der Schlussfolgerung: „Letztlich erfordert die Selbststeuerung eine Komplementarität auf der Organisationsebene, die sich selbst veränderungsoffen, selbstkritisch und lernfreudig zeigen muss" (S. 9).

Exemplarisch soll nachfolgend die Möglichkeit einer Lernkulturanalyse als kontinuierliche Massnahme zur Organisationsentwicklung erläutert werden, um systematisch erforderliche Rahmenbedingungen für Lernen und Innovationsprozesse zu unterstützen.

\subsubsection{Lernkulturanalyse als kontinuierliche OE Maßnahme}

Eine systematische Analyse der bestehenden Lernkultur ermöglicht eine Standortbestimmung hinsichtlich von Gestaltungsbereichen, die für die Lern- und Innovationsfähigkeit einer Organisation zentral sind. Im Rahmen der Lernkulturanalyse (vgl. u.a. Seufert, Hasanbegovic \& Euler, 2007) werden fünf Aspekte von Lernkultur über validierte Fragebögen jeweils separat für Mitarbeitende und für Führungskräfte ermittelt (s. auch Abschnitt 4.4.1):

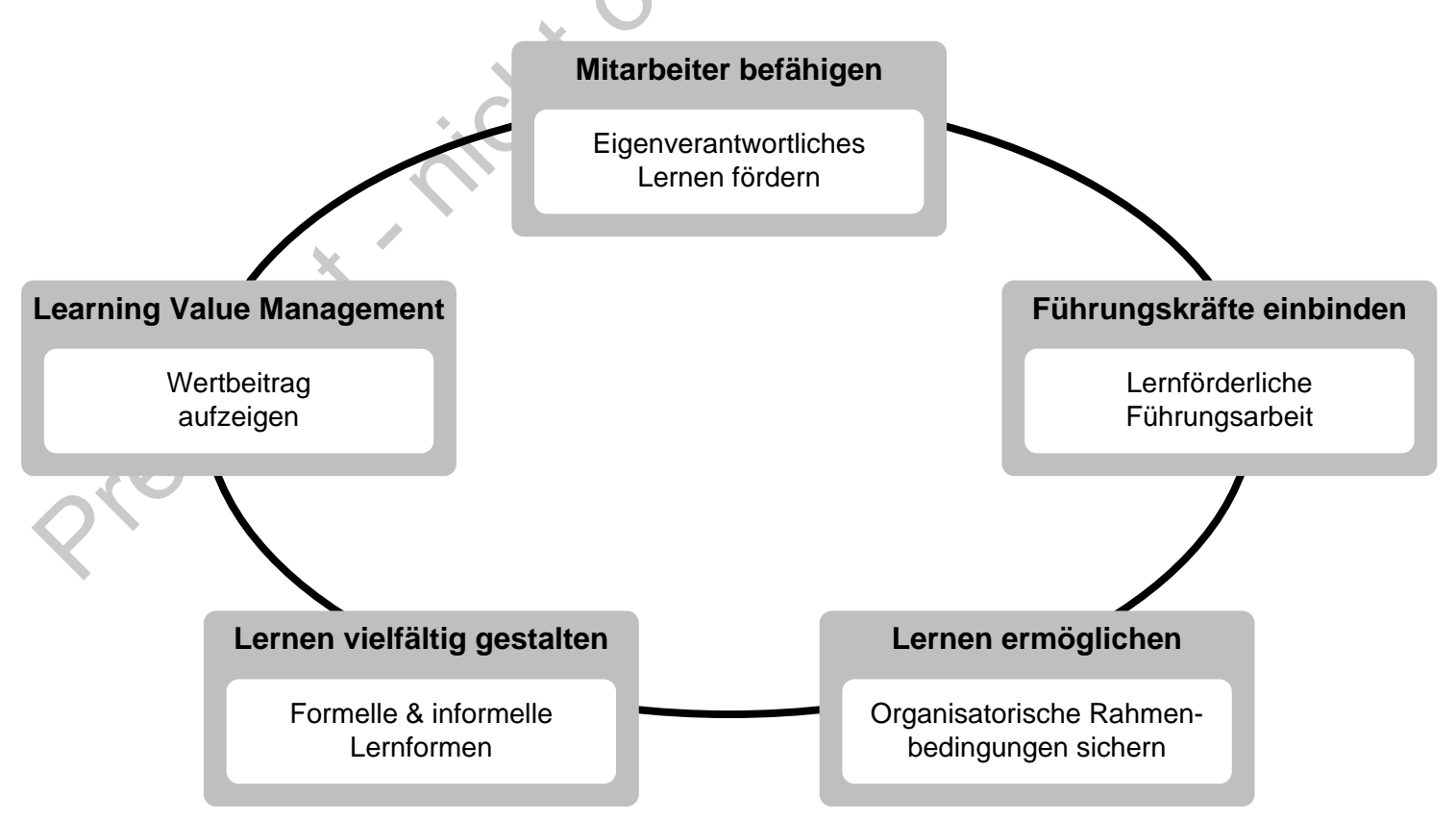

Abbildung 10: Instrument zur Erhebung der Lernkultur als Ausgangspunkt für Maßnahmen zur Organisationsentwicklung (in Anlehnung an Seufert et al., 2007, S. 19) 
Die Durchführung einer solchen Lernkulturanalyse gibt nicht nur Auskunft über den Status Quo in einem bestimmten Unternehmen resp. einer Organisation und den Stand im Vergleich zu anderen. Die Ergebnisse liefern Hinweise darauf, konkrete Maßnahmen zu initiieren, um mögliche Lern- und Innovationsbarrieren abzubauen oder akzeptanzfördernde Supportmaßnahmen zu lancieren.
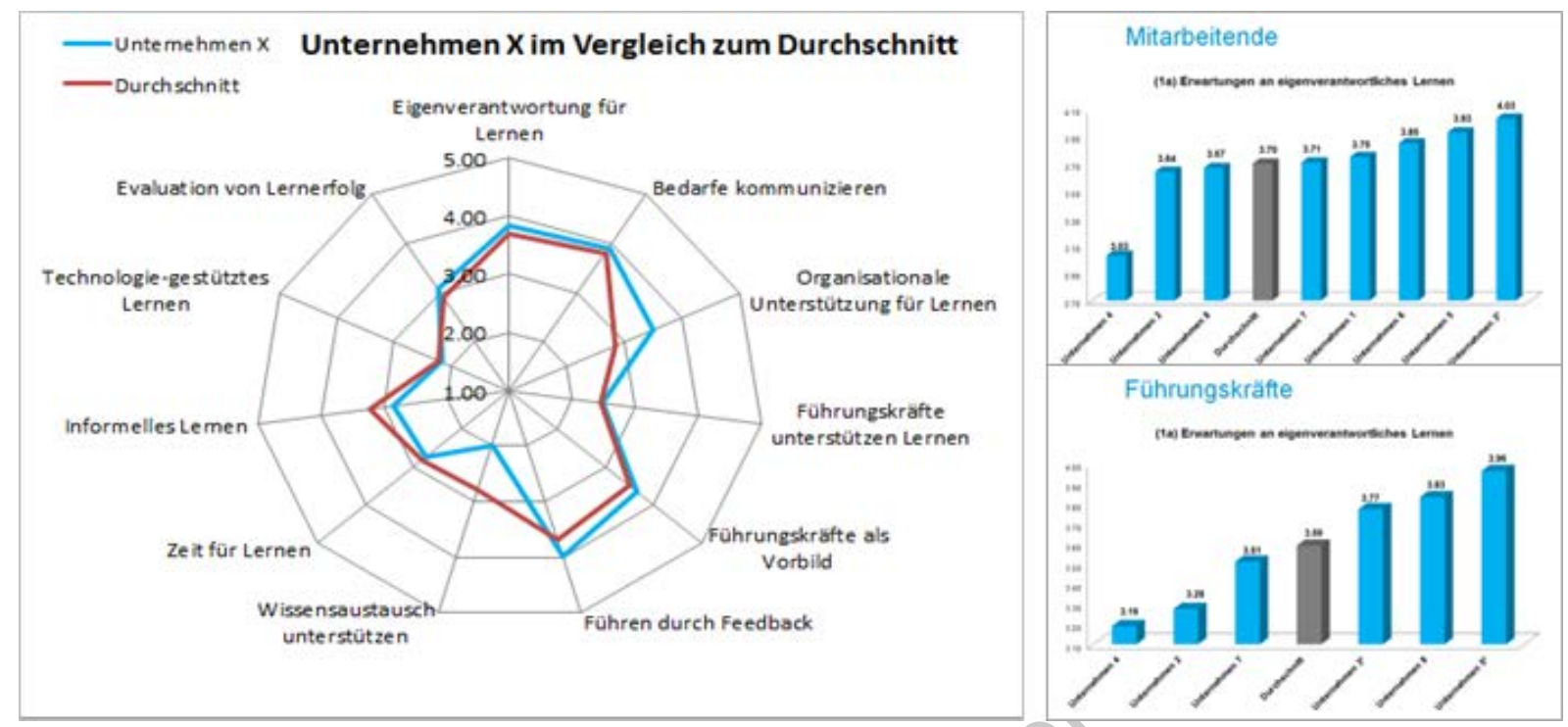

Abbildung 11: Beispiele für Benchmarking-Ergebnisse im Rahmen einer Lernkulturanalyse

Standortbestimmung und Benchmarking: Die Ergebnisse der Lernkultur-Analyse können den Ausgangspunkt bieten für

- $\quad$ eine interne Diskussion zum Thema Lernen (Zukunftsbild, Rollen, Verantwortlichkeiten),

- die Identifikation neuer Handlungsbereiche und Maßnahmen,

- ein neues/verändertes Learning Design,

- eine veränderte Rolle von Führungskräften im Lernen,

- $\quad$ eine Veränderung der Rahmenbedingungen (z.B. Arbeitszeit, Räumlichkeiten, etc.),

- $\quad$ eine stärkere Verknüpfung formeller und informeller Lernprozesse,

- $\quad$ neue Vorgehensweise im Aufzeigen des Wertbeitrags von Bildungsmaßnahmen.

Im Rahmen der Lernkultur-Analyse kann auch ein Benchmarking zwischen verschiedenen Abteilungen oder Bildungsbereichen von Netzwerk-Organisationen von Nutzen sein. Die BenchmarkMethode von Robert Camp erachtet die Identifikation und Implementierung von Best Practices als zentrale Aufgaben (Camp, 1995; Camp \& Steinhoff, 1994). Benchmarking stellt einen der effektivsten Wege dar, externes Wissen rasch in das eigene Unternehmen einzubringen, da es aus der Praxis für die Praxis erhoben wird und damit im höchsten Masse anwendungsorientiert ist. Dabei liegt die Annahme vor, dass ein Benchmarking nicht dazu führen sollte, Bestleistungen von Konkurrenten in der Branche nachzuahmen, sondern zunächst ein Verständnis für erfolgreiche Strategien zu entwickeln, um dann vielmehr eigene Wege wählen zu können („Kapieren statt kopieren“).

Ziel des vorgestellten Analyse- und Gestaltungsrahmens von Lernkulturen ist es folglich, aus Sicht des Bildungsmanagements relevante Merkmale von Lernkulturen zu erfassen und damit die Basis für die Ableitung von Gestaltungsempfehlungen zur Optimierung nachhaltigen Lernens in Organisationen zu schaffen. Diese Dimensionen verdeutlichen, dass strukturelle Faktoren im Vordergrund stehen, welche lernförderliche Rahmenbedingungen in einer Organisation darstellen und somit die von den Mitarbeitenden und Führungskräften wahrgenommene Kultur maßgeblich mit beeinflussen können. Im Unterschied zu existierenden Diagnoseinstrumenten zur lernenden Organisation (siehe Tabelle 6) oder lernkulturellen Merkmalen einer Organisation, kann insbesondere mit diesem Modell eine Brücke zwischen Organisations- und Personal-/ Kompetenzentwicklung geschlagen werden. Die Dimensionen 
und Indikatoren zur Präzisierung des Konstrukts Lernkultur knüpfen somit direkt an den Verantwortungs- und Gestaltungsbereichen des Bildungsmanagements in einer Unternehmung an.

\section{Zusammenfassung}

Die Innovationsfähigkeit von Unternehmen ist untrennbar mit Lernen verbunden. Erst ein Lernen auf einem höheren Niveau ermöglicht es, Innovationen umzusetzen, indem nicht nur Anpassungslernen, sondern Prozesslernen (Deutero Learning) stattfindet. Dies bezieht sich vor allem auf zwei Ebenen: Die individuelle und die organisationale. Führen dann persönlich oder auch organisational verankerte Barrieren dazu, dass Prozesslernen gehemmt wird, wirkt sich das ebenso negativ auf die Innovationsfähigkeit aus. Daher ist es bedeutend, solche Hindernisse zu überwinden. Für die Steigerung der Lern- und Innovationsfähigkeit ist auf der Basis einer systemischen Betrachtungsweise das Verhalten des Systems sowie die Wechselwirkungen innerhalb dessen zu verstehen und zu steuern. Das hier vorgestellte Rahmenkonzept fokussiert das Ziel, die Lern- und Innovationsfähigkeit zu steigern, wozu eine stärkere Verzahnung von Personal- und Organisationsentwicklung grundlegend ist. Es stellt die Organisation in Verbindung zu ihrer Strategie, ihren Strukturen und Kulturen, auf deren Grundlage vier Handlungsfelder vorgestellt werden: Die Förderung individuellen innovativen Verhaltens durch Kompetenzentwicklung, lern- und innovationsorientierte Führungskräfteentwicklung, Gestaltung innovativer Arbeits- und Vernetzungsformen sowie lern- und innovationsförderlicher Rahmenbedingungen.

In Rückbezug auf das Business Innovation Modell lässt sich festhalten, dass die bewusste und lernförderliche Gestaltung der vier Handlungsfelder mit dem übergeordneten Ziel der Verzahnung von Personal- und Organisationsentwicklung einen bedeutenden Teil zur Verankerung der Business Innovation im Unternehmen beiträgt.

\section{Literaturverzeichnis}

Argyris, C. \& Schön, D. A. (1999). Die lernende Organisation. Grundlagen, Methode, Praxis. Stuttgart: Klett-Cotta.

Bass, B. M. \& Stogdill, R. M. (1990). Bass \& Stogdill's handbook of leadership. Theory, research, and managerial applications (3rd ed). New York (N.Y.): Free Press [etc.].

BMBF. (2007). Arbeiten - Lernen - Kompetenzen entwickeln. Innovationsfähigkeit in einer modernen Arbeitswelt. Verfügbar unter http://www.arbeitsinnovation.de/common/download/Arbeit-LernenKompetenzen_2007-2011.pdf

Böhle, F., Pfeiffer, S. \& Sevay-Tegethoff, N. (2004). Die Bewältigung des Unplanbaren. Wiesbaden: Verlag für Sozialwissenschaften.

Bruch, H., Krummacher, S. \& Vogel, B. (op. 2012). Leadership - Best Practices und Trends (2., aktualisierte und erw. Aufl). Wiesbaden: Gabler.

Camp, R. C. \& Steinhoff, A. (1994). Benchmarking. München: Hanser.

Camp, R. C. (1995). Business process benchmarking finding and implementing best practices. Milwaukee: ASQC Quality Press.

Dehnbostel, P. \& Pätzold, G. (2004). Lernförderliche Arbeitsgestaltung und die Neuorientierung betrieblicher Bildungsarbeit. In P. Dehnbostel \& G. Pätzold (Hrsg.), Innovationen und Tendenzen der betrieblichen Berufsbildung (Beiheft 18, S. 19-30). Stuttgart: Franz Steiner.

Dietrich, S. (Hrsg.). (2001). Selbstgesteuertes Lernen in der Weiterbildungspraxis. Ergebnisse und Erfahrungen aus dem Projekt SeGeL. Bielefeld: Bertelsmann. 
Ekvall, G. (1996). Organizational climate for creativity and innovation. European Journal of Work and Organizational Psychology, 5, 105-123.

Erpenbeck, J. (2004). Erfahrungswerte - Werterfahrung. Nichtimitierbarkeit und berufliche Bildung. In P. Dehnbostel \& G. Pätzold (Hrsg.), Innovationen und Tendenzen der betrieblichen Berufsbildung (Beiheft 18, S. 43-54). Stuttgart: Franz Steiner.

Geyer, A. \& Steyrer, J. (1998). Messung und Erfolgswirksamkeit transformationaler Führung. Zeitschrift für Personalforschung, 12 (4), 377-401.

Gibb, S. (2003). Line manager involvement in learning and development: Small beer or big deal? Employee Relations, 3 (25), 281-293.

Göhring, M. (2008). Banken und Kommunikation 2.0. In D. Spath, W. Bauer \& M. Engstler (Hrsg.), Innovationen und Konzepte für die Bank der Zukunft. Mit modernen Vertriebslösungen und optimierten Wertschöpfungsprozessen künftigen Herausforderungen begegnen (1. Aufl.). Wiesbaden: Gabler. Verfügbar unter http://link.springer.com/content/pdf/10.1007\%2F978-3-83499880-4_18.pdf

Haarich, M., Sparschuh, S., Zettel, C. \& Hees, F. (2011). Innovationsfähigkeit - Lernfähigkeit Transferfähigkeit. Innovationen systematisch fördern. In S. Jeschke, I. Isenhardt, F. Hees \& S. Trantow (Hrsg.), Enabling Innovation. Innovationsfähigkeit - deutsche und internationale Perspektiven (SpringerLink : Bücher, S. 447-464). Berlin, Heidelberg: Springer-Verlag Berlin Heidelberg.

Haller, C. (2003). Verhaltenstheoretischer Ansatz für ein Management von Innovationsprozessen. Universität Stuttgart (Dissertation).

Hart, J. (2013). The Workplace Learning Revolution. Free mini e-Book (2. Aufl.). Verfügbar unter http://de.slideshare.net/janehart/the-workplace-learning-revolution

Hartmann, D., Brentel, H. \& Rohn, H. (2006). Lern- und Innovationsfaehigkeit von Unternehmen und Organisationen. Kriterien und Indikatoren. Wuppertal Paper: 156. Zugriff am 07.11.2013. Verfügbar unter http://www.econstor.eu/bitstream/10419/29716/1/511648723.pdf

Kauffeld, S., Jonas, E., Grote, S., Frey, D. \& Frieling, E. (2004). Innovationsklima - Konstruktion und erste psychometrische Überprüfung eines Messinstruments. Diagnostica, 50, 153-164.

Koch, M., Richter, A. \& Stocker, A. (2012). Enterprise 2.0 - Wissensmanagement der neuen Generation? Zugriff am 24.08.2013. Verfügbar unter http://www.soziotech.org/enterprise-2-0wissensmanagement-der-neuen-generation/

Lang, M. \& Pätzold, G. (2004). Neue Wege zum Erwerb von Arbeitsprozesswissen in hochautomatisierten Produktionsprozessen der Chemischen Industrie - Die Eignung netzbasierter Lernumgebungen zur Verknüpfung von formellem und informellem Lernen im Arbeitsprozess. In $P$. Dehnbostel \& G. Pätzold (Hrsg.), Innovationen und Tendenzen der betrieblichen Berufsbildung (Beiheft 18, S. 97-106). Stuttgart: Franz Steiner.

Manning, S. (2002). Resource base of a research project cluster related to human resource development in Europe. Berlin: WIFO Research Forum.

Meiser, K. (2001). Vorbemerkungen. In S. Dietrich (Hrsg.), Selbstgesteuertes Lernen in der Weiterbildungspraxis. Ergebnisse und Erfahrungen aus dem Projekt SeGeL (S. 7-9). Bielefeld: Bertelsmann.

Müller-Stewens, G. \& Lechner, C. (2005). Strategisches Management. Wie strategische Initiativen zum Wandel führen (3. Aufl.). Stuttgart: Schäffer-Poeschel.

Osterloh, M. (2001). Wettbewerbsfähigkeit in der Wissensgesellschaft oder: Können Organisationen lernen? In QUEM (Hrsg.), Arbeiten und Lernen. Lernkultur Kompetenzentwicklung und innovative Arbeitsgestaltung (S. 123ff). Berlin: QUEM. 
Pedler, M., Burgoyne, J. \& Boydell, T. (1991). The learning company. A strategy for sustainable development. London: McGraw-Hill.

Reinmann, G. (2013). Reader zum Thema entwicklungsorientierte Bildungsforschung. Zugriff am 29.10.2013. Verfügbar unter http://gabi-reinmann.de/wpcontent/uploads/2013/01/Reader_Entwicklungsforschung_Jan2013.pdf

Rüegg-Stürm, J. (1998). Entwicklung und Messbarkeit von Wandelfähigkeit. Stolpersteine und Perspektiven der Lernenden Organisation. In Eidgenössisches Personalamt (Hrsg.), Wie die Verwaltung lernt. Der öffentliche Sektor auf dem Weg zur lernenden Organisation (Schriftenreihe des Eidgenössischen Personalamtes, Bd. 11, S. 239-262). Bern: Eidgenössische Drucksachenund Materialzentrale.

Rüegg-Stürm, J. (2003). Das neue St. Galler Management-Modell. Grundkategorien einer integrierten Managementlehre ; der HSG-Ansatz (2., durchges. Aufl). Bern [u.a.]: Haupt.

Salazar, Y. \& Peters, S. (2011). Betriebliches Lernen in Händen neuer Akteure - Herausforderungen für die Innovationsfähigkeit von Unternehmen. In S. Jeschke, I. Isenhardt, F. Hees \& S. Trantow (Hrsg.), Enabling Innovation. Innovationsfähigkeit - deutsche und internationale Perspektiven (SpringerLink : Bücher, S. 127-138). Berlin, Heidelberg: Springer-Verlag Berlin Heidelberg.

Schuchmann, D. \& Seufert, S. (2013). Neuorientierung betrieblicher Weiterbildung. In S. Seufert \& C. Metzger (Hrsg.), Kompetenzentwicklung in unterschiedlichen Lernkulturen. Festschrift für Dieter Euler zum 60. Geburtstag (1. Aufl. 2013, S. 421-442). Paderborn: Eusl.

Seibold, P. (1995). Noch Platz für Organisatoren in der lernenden Bank? In Congena (Hrsg.), Die lernende Bankorganisation. Strategien für die Jahrtausendwende (S. 193-204). Wiesbaden: Gabler.

Senge, P. M. (1996). Die fünfte Disziplin. Stuttgart: Klett-Cotta.

Seufert, S., Hasanbegovic, J. \& Euler, D. (2007). Mehrwert für das Bildungsmanagement durch nachhaltige Lernkulturen (Scil Arbeitsbericht 11). St. Gallen: Institut für Wirtschaftspädagogik.

Seufert, S. (2011). Informelles Lernen. Wie Sie mit Social Media eine innovative Lernkultur schaffen. Zeitschrift für Organisationsentwicklung, 5 (80), 299-305.

Seufert, S. (2012). Die digitale Revolution und die Evolution des Lehrens. Folio, 4, 36-38.

Seufert, S. (2013). Bildungsmanagement. Einführung für Studium und Praxis. Stuttgart: SchäfferPoeschel.

Sonntag, K., Schaper, N. \& Friebe, J. (2007). Erfassung und Bewertung von Merkmalen unternehmensbezogener Lernkulturen. In Arbeitsgemeinschaft QUEM (Hrsg.), Kompetenzmessung im Unternehmen, Lernkultur- und Kompetenzanalysen im betrieblichen Umfeld (QUEM-Report, Bd. 18, S. 19-340). Berlin: Waxmann.

Stobbe, A. (2010). Enterprise 2.0 - Wie Unternehmen das Web 2.0 für sich nutzen (Deutsche Bank Research, Hrsg.), Frankfurt a. M.

Wahren, H.-K. E. (1996). Das lernende Unternehmen. Theorie und Praxis des organisationalen Lernens : [mit Tabellen]. Berlin [u.a.]: de Gruyter.

Weissenberger-Eibl, M. (2010). Innovationen und Lernen. Mündlicher Vortrag auf dem F.PAK Kolloquium in München. Verfügbar unter www.applied-knowing.org/de/fpak/?news=168

Wenger, E. (1998). Communities of Practice: Learning, Meaning, and Identity. Cambridge: Cambridge University Press.

Wilkesmann, U. (1999). Lernen in Organisationen. Die Inszenierung von kollektiven Lernprozessen (Campus Forschung, Bd. 782, ). Frankfurt/Main [u.a.]: Campus-Verl. 
Wunderer, R. (2003). Führung und Zusammenarbeit. Eine unternehmerische Führungslehre (5., überarb. Aufl). Köln: Luchterhand in Wolters Kluwer Deutschland.

Yang, B., Watkins, K. \& Marsick, V. J. (2004). The construct of the learning organization: Dimensions, measurement, and validation. Human Resource Development Quarterly, 15 (1), 31-55. 